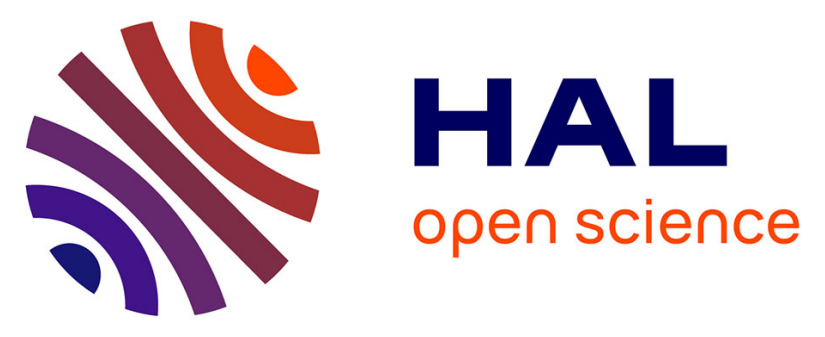

\title{
Evolution of microparasites in spatially and genetically structured host populations: The example of RHDV infecting rabbits
}

David Fouchet, Jacques Le Pendu, J.-S. Jean-Sébastien Guitton, Micheline Guiserix, S. Stéphane Marchandeau, Dominique Pontier

\section{To cite this version:}

David Fouchet, Jacques Le Pendu, J.-S. Jean-Sébastien Guitton, Micheline Guiserix, S. Stéphane Marchandeau, et al.. Evolution of microparasites in spatially and genetically structured host populations: The example of RHDV infecting rabbits. Journal of Theoretical Biology, 2009, 257 (2), pp.212. 10.1016/j.jtbi.2008.11.021 . hal-00554541

\section{HAL Id: hal-00554541 https://hal.science/hal-00554541}

Submitted on 11 Jan 2011

HAL is a multi-disciplinary open access archive for the deposit and dissemination of scientific research documents, whether they are published or not. The documents may come from teaching and research institutions in France or abroad, or from public or private research centers.
L'archive ouverte pluridisciplinaire HAL, est destinée au dépôt et à la diffusion de documents scientifiques de niveau recherche, publiés ou non, émanant des établissements d'enseignement et de recherche français ou étrangers, des laboratoires publics ou privés. 


\section{Author's Accepted Manuscript}

Evolution of microparasites in spatially and genetically structured host populations: The example of RHDV infecting rabbits

David Fouchet, Jacques Le Pendu, Jean-Sébastien Guitton, Micheline Guiserix, Stéphane Marchandeau, Dominique Pontier

PII: S0022-5193(08)00608-5

DOI: doi:10.1016/j.jtbi.2008.11.021

Reference: $\quad$ YJTBI5373

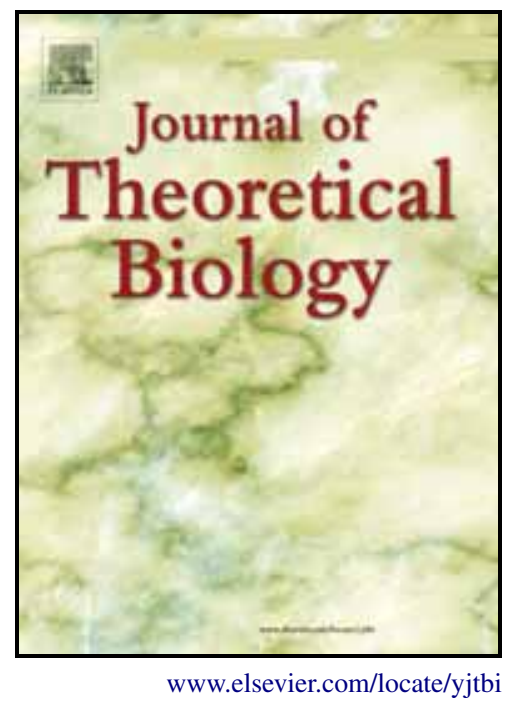

To appear in: $\quad$ Journal of Theoretical Biology

Received date: $\quad 30$ April 2008

Revised date: $\quad 19$ November 2008

Accepted date: 19 November 2008

Cite this article as: David Fouchet, Jacques Le Pendu, Jean-Sébastien Guitton, Micheline Guiserix, Stéphane Marchandeau and Dominique Pontier, Evolution of microparasites in spatially and genetically structured host populations: The example of RHDV infecting rabbits, Journal of Theoretical Biology (2008), doi:10.1016/j.jtbi.2008.11.021

This is a PDF file of an unedited manuscript that has been accepted for publication. As a service to our customers we are providing this early version of the manuscript. The manuscript will undergo copyediting, typesetting, and review of the resulting galley proof before it is published in its final citable form. Please note that during the production process errors may be discovered which could affect the content, and all legal disclaimers that apply to the journal pertain. 


\section{Evolution of microparasites in spatially and genetically structured host populations: the example of RHDV infecting rabbits}

David Fouchet ${ }^{\mathrm{a},{ }^{*}}$, Jacques Le Pendu ${ }^{\mathrm{b}}$, Jean-Sébastien Guitton ${ }^{\mathrm{c}}$, Micheline Guiserix ${ }^{\mathrm{a}}$, Stéphane Marchandeau $^{\mathrm{c}}$, Dominique Pontier ${ }^{\mathrm{a}}$

${ }^{a}$ UMR C.N.R.S. 5558 "Biométrie et Biologie Evolutive"; Université de Lyon ; Université

Lyon1 ; 43 Boul. 11 Novembre 1918, 69622 Villeurbanne Cedex, France

fouchet@biomserv.univ-lyon1.fr, guiserix@biomserv.univ-lyon1.fr, dpontier@biomserv.univ-lyon1.fr

${ }^{b}$ INSERM U601, Université de Nantes, 44093, Nantes, France.

jlependu@nantes.inserm.fr

${ }^{c}$ Office National de la Chasse et de la Faune Sauvage, 44000 Nantes, France.

jean-sebastien.guitton@oncfs.gouv.fr, stephane.marchandeau@oncfs.gouv.fr

${ }^{*}$ Corresponding author

UMR-CNRS 5558 ; Université de Lyon ; Université Lyon1 ; 43 Boulevard du 11 Novembre 1918 ; 69622 Villeurbanne Cedex, France.

E-mail: fouchet@,biomserv.univ-lyon1.fr

Tel:(33)4-72-43-13-37

Fax:(33)4-72-43-13-88 


\section{$\underline{\text { Abstract }}$}

Several studies have shown that classical results of microparasite evolution could not extend to the case where the host species shows an important spatial structure. Rabbit Haemorrhagic Disease Virus (RHDV), responsible for Rabbit Haemorrhagic Disease (RHD), which recently emerged in rabbits, has strains within a wide range of virulence, thus providing an interesting example of competition between strains infecting a host species with a metapopulation structure. In addition, rabbits may show a genetic diversity regarding RHDV susceptibility. In the present paper we use the example of the rabbit-RHDV interaction to study the competition between strains of a same microparasite in a host population that is both spatially and genetically structured. Using metapopulation models we show that the evolution of the microparasite is guided by a trade-off between its capacity to invade subpopulations potentially infected by other strains and its capacity to persist within the subpopulation. In such a context, host genetic diversity acts by reducing the number of hosts susceptible to each strain, often favouring more persistent - and generally less virulent - strains. We also show that even in a stochastic context where host genes regularly go locally extinct, the microparasite pressure helps maintain the genetic diversity in the long term while reinforcing gene loss risk in the short term. Finally, we study how different demographic and epidemiologic parameters affect the coevolution between the rabbit and RHDV.

Keywords: Evolution of virulence, metapopulation, stochastic model, Matching-Allele model 


\section{Introduction}

Microparasites are even more affected by natural selection than other organisms, due to their short life cycle. For a given microparasite, strains differing in pathogenicity, in particular, compete with each other for resources, i.e. the host. The severity of the disease resulting from the infection is thus highly influenced by natural selection. Understanding the factors influencing the evolution of microparasites is thus crucial to assess the impact of the associated diseases (Dieckmann et al., 2002).

For the last two decades, one theory has predominated for understanding the evolution of microparasites without co- or super-infection. This theory predicts that the competition between two strains of a same parasite is won by the strain with the highest basic reproductive number $R_{0}$ (Anderson and May, 1979; May and Anderson, 1983), where $R_{0}$ can be interpreted as the mean number of secondary cases induced by a single infected individual released in a wholly susceptible host population during its infectious period.

Beyond the highlights brought by the $R_{0}$ theory in the understanding of microparasite evolution, it fails to predict the evolution of parasite virulence in many circumstances (see Dieckmann et al., 2002). A large set of works has challenged this theory in spatially structured host populations. In contact networks, several theoretical studies (Boots and Sasaki, 1999; Boots et al., 2004; Claessen and de Roos, 1995; Rand et al., 1995) have found that strains having a high basic reproductive number tend to be surrounded by either dead or recovered individuals if they exploit their host too fast, leading to their long-term extinction and to the selection of strains that exploit their host slower even if they have lower basic reproductive numbers. The idea that more localised parasitic exchanges between individuals tend to select for less transmissible strains has been recently challenged experimentally with success (Boots and Mealor, 2007). In addition, in hosts structured into a metapopulation, Keeling (2000) has demonstrated that, especially in highly stochastic and poorly connected 
sets of subpopulations, the ability of a strain to persist at a local scale could be more important than its local competition characteristics $\left(R_{0}\right)$. However, the model is population-based, and the validity of such approaches needs to be tested by more explicit models (Keeling, 2002).

Another aspect of many host-parasite interactions is that the host genetic diversity often leads to variable susceptibility of individuals to the different strains. Experimental and theoretical works suggest a negative relationship between the genetic diversity of a host population and the impact of some of its pathogens (Alitzer et al., 2006; Hughes and Boomsma, 2004; O'Brien and Evermann, 1988; Pearman and Garner, 2005; Siemens and Roy, 2005). One well known mechanism for that is that genetic diversity reduces the transmission potential of the pathogen by reducing the density of susceptible hosts hence leading to decreased disease prevalence. However, little attention has been paid to the long term evolutionary consequences of the host polymorphism on the microparasite virulence in a spatial context.

Due to their particular spatial and social organisation, European rabbits (Oryctolagus cuniculus) provide a valuable example of a host species in which the theory of the $R_{0}$ could fail to predict the evolution of viruses whose most locally competitive strains are not the most persistent ones at the scale of the rabbit subpopulation. RHDV (Rabbit Haemorrhagic Disease Virus) is a lagovirus belonging to the Caliciviridae and responsible for the Rabbit Haemorrhagic Disease (RHD). It has been identified for the first time in China in 1984 (Liu et al., 1984) where it killed 140 millions of farmed rabbits. However, retrospective studies demonstrated that the virus was present worldwide, decades before the local emergence of the symptomatic infection (Moss et al., 2002; Rodak et al., 1990). RHDV strains isolated in the field are generally highly lethal (up to $95 \%$ mortality 2 days post-infection) and transmissible, but interestingly the impact of the virus has been highly contrasted. Some populations have a high proportion of seropositive rabbits in the absence of recorded mass mortality due to the 
disease (Chasey et al., 1995; Cooke et al., 2000; Marchandeau and Boucraut-Baralon, 1999; Marchandeau et al., 1998; Rodak et al., 1990; Sanson et al., 2000; Trout et al., 1997), suggesting that strains of RHDV of different virulence circulate in the field even if such strains have not yet been isolated. A non-pathogenic strain was isolated from an Italian rabbitry (Capucci et al., 1996), and it may be the cause of the seroprevalence-withoutmortality seen in the field. However, a study in the UK failed to find it (Moss et al., 2002), suggesting that other strains causing zero (or low) mortality exist. During infection by avirulent strains, virions are hardly detectable (Capucci et al., 1996), suggesting that they are poorly transmissible. To explain their persistence, it has been suggested that they could induce a long lasting, and even lifelong, infection (White et al., 2001), which balances their low level of transmission.

In addition, rabbits may show genetic diversity in their susceptibility to RHDV. Recent work has shown that some rabbits are resistant to the infection (Le Pendu, unpublished results). The rabbit-RHDV system can be paralleled with that of noroviruses, another genus from the Caliciviridae family causing gastro-enteretis in humans. It has been shown that the susceptibility or resistance to noroviruses depends on the presence of fucoses on the surface of epithelial cells, under the control of a polymorphic genetic system (Marionneau et al., 2001). As a result, most strains of the virus can only infect a given genetic subset of the human population (Le Pendu et al., 2006; Tan and Jiang, 2005). The presence of fucoses is also required for binding a highly pathogenic RHDV strain to rabbit epithelial cells (RuvoënClouet et al., 2000) and recent observations suggest the existence of two phenotypes for rabbits: secretors, which present fucoses, and non-secretors, which do not. The former are expected to be susceptible to RHDV while the latter are expected to be resistant (unpublished results). 
Until recently, non-secretors were under-represented in rabbit populations, but our unpublished preliminary results suggest that their resistance to RHDV has led to their rapid increase. Given the emergence of this large population still uninfected by the virus and considering the ability of the virus to produce viable mutants on a short ecological timescale the analysis of RHDV stains circulating in France between 1993 and 2000 shows a large degree of variation in RHDV strains (Le Gall-Reculé et al., 2003) -, the virus could find an alternative way to bind cells of non-secretors, as noroviruses did in humans. So it seems plausible that mutants infecting non-secretor rabbits could emerge and in this case would rapidly invade. Just like for noroviruses in which most of the strains are unable to infect both secretors and non-secretors (Le Pendu et al., 2006; Tan and Jiang, 2005), the RHDV would thus fit the Matching-Allele (MA) model. This relation is still speculative for rabbits, but the Matching allele model is largely used to assess general issue in host parasite coevolution theories (Hamilton et al., 1990; Howard and Lively, 1994), so the scope of this study goes beyond the simple analysis of the rabbit-RHDV system.

One key feature of MA models is that the fitness of a host depends on the frequency of its phenotype: when a phenotype is less represented, the corresponding pathogen strain can hardly spread, so it suffers less parasite circulation and thus less mortality (Bell and Maynard Smith, 1987; Clarke, 1976; Hamilton, 1980; Hamilton et al., 1990). This favours the maintenance of genetic diversity.

In the present paper we build a mathematical model to investigate how the rabbit population structure may affect the long-term evolution of RHDV. In a first step, we focus on the effect of the spatial structure on the virus evolution assuming a classical trade-off between strains' transmission and the duration of the infection they induce, without genetic structure of the rabbit population. In a second step, we study how the genetic diversity of rabbits could 
affect the results on evolution of RHDV obtained previously. Finally, we investigate the effect of the different model parameters on the stability of the polymorphism.

\section{1) Material and methods}

a. Virus genetic diversity

Until now, two different kinds of strains have been isolated in the field. The virulent one is the most spectacular (due to its high lethality) and is the most often found in the field. The avirulent strain has been rarely isolated (Capucci et al., 1996), but its presence is suspected in many field studies (Chasey et al., 1995; Cooke et al., 2000; Marchandeau and BoucrautBaralon, 1999; Marchandeau et al., 1998; Rodak et al., 1990; Sanson et al., 2000; Trout et al., 1997). This can be explained by a likely sampling bias, since rabbit populations have been mostly sampled after an outbreak of RHD mortality.

As seen previously, it is reasonable to assume that the two kinds of strains use two opposite strategies. Virulent strains are highly transmissible but induce short duration diseases (in most cases the rabbit dies within 2 days). Avirulent strains are poorly transmissible but induce long lasting (or even chronic, see White et al., 2001) infections. In addition, it is also reasonable to assume that virulent strains have higher $R_{0}$. If virulent strains had a lower basic reproductive number than avirulent ones, it would prevent them from invading and the shortlasting infections they induce would lead them to a rapid extinction. This phenomenon has been observed in myxoma-virus for which highly virulent strains have been excluded because of their lower $R_{0}$ than less virulent strains (Dwyer et al., 1990; Levin and Pimentel, 1981).

RHDV is an interesting example of a parasite facing the conflict between local adaptation and local persistence (Keeling, 2000). Unfortunately, the characteristics of the different strains are still unknown. What is the relative transmission rate between virulent and avirulent strains? How long can avirulent strains be transmitted in the field? Do strains with 
intermediate characteristics exist? Since no definitive answer can be given to these questions, this paper must to some extent remain conceptual. How does the rabbit population structure influence the evolution of RHDV? This is basically the kind of question that we can address now. More quantitaive questions would require more precise knowledge about RHDV strain characteristics.

Among the simplifications made in the model, we assume the existence of strains of intermediate virulence. Such a quasi continuum can be observed in other host-parasite interactions (e.g. in myxomatosis, Fenner, 1953). Intermediate virulence strains have not been isolated yet for RHDV, but this does not prove that they do not exist. Since most attention has focused until now on highly virulent strains, strains of reduced virulence are rarely studied. Assuming this quasi continuum is more convenient for studying the impact of the parameters on the evolution of RHDV. Small changes in the host-parasite characteristics will slightly increase the strain of the continuum which is the most favoured. The effects of the same changes in the parameters can remain undetected with a two strains model, because the same strain can be selected in large regions of the parameters. Removing the hypothesis of a continuum would not much alter the qualitative conclusions of the paper.

In the next sections we will often refer to the persistence of a strain. The term persistence can define three different things: persistence within the host (i.e. duration of the disease), persistence within one rabbit subpopulation (the "local persistence") and persistence within the metapopulation (the "global persistence"). Clearly these three notions are linked. Strains inducing a longer disease will tend to persist longer within the subpopulation and hence within the metapopulation. However this association is not systematic. For example, even a strain inducing a lifelong disease can persist poorly locally if it has a very low transmission rate. 
Finally strains can also differ in the rabbit phenotype they are able to infect. In a first step we assume that all strains are able to infect all non-infected and non-recovered rabbits equally: rabbits are not genetically differentiated. We define this model as the model without host genetic diversity. Next we assume the existence of two phenotypes for rabbits: secretors $(\boldsymbol{S E})$ and non-secretors $(\boldsymbol{s e})$. Each RHDV strain is then specialized: it can infect only one phenotypic group of rabbits (model with host genetic diversity).

In the next two sections we present the deterministic and stochastic mathematical models we developed for evaluating the effect of the host demographic characteristics on the evolution of the virus and its ability to promote the host genetic polymorphism.

\section{b. Model without host genetic heterogeneity}

The deterministic model extends the classical SIR model with continuous time. The population is divided into three compartments: susceptible $S$, infected $I$ and recovered $R$, with $N=S+I+R$ the size of the host population. Different models have been proposed for the transmission rate of the virus: the proportionate mixing model $\beta . S . I / N$ (White et al., 2001) which assumes that the contact rate between rabbits does not depend on the number of rabbits within the population, and the mass action law $\beta . S . I$ (Calvete, 2006) where the contact rate between rabbits linearly increases with the rabbit population size. Here for the sake of simplicity we consider only the mass action law (Anderson and May, 1981). However, the main results of the model, i.e. those obtained with the stochastic versions of the model, remain qualitatively true with the alternative proportionate mixing model (see electronic supplementary materials).

Infected individuals die at the rate $\alpha$ or recover at the rate $\sigma$. After recovery they become lifelong protected. All young are born susceptible (there is no vertical transmission) and $b$ represents the birth rate of the host population. The natural death rate, i.e. from any 
other cause than RHDV (e.g., predation, other parasites), is chosen density-dependent, $\mu_{0}+\delta N$, in order to maintain the disease-free population at its limit size $K=\left(b-\mu_{0}\right) / \delta$, called carrying capacity.

Now we extend the model to consider several strains of RHDV and the metapopulation structure of the host population (Figure 1).

Modelling the RHDV strains. To model different levels of pathogenicity of RHDV strains, we split the $I$ compartment into $N_{V}$ compartments, corresponding to $N_{V}$ different levels of pathogenicity of the virus. Furthermore, we assume that when a rabbit is infected or has recovered it cannot be reinfected by any other strain (no co-infection, no super-infection and total and lifelong cross-immunity). Strains are ranked according to their transmission rate $\beta_{i}$ $\left(i=1 . . N_{V}\right) . \beta_{0}$ describes the transmission rate of the virulent strain (strain $\left.N_{V}\right)$. The transmission rate increases geometrically from strain $1\left(\beta_{I}=2.5 \times 10^{-4} \beta_{0}\right)$ to strain $N_{V}\left(\beta_{N_{V}}=\beta_{0}\right) . I_{i}$ represents the number of individuals infected by a strain of $i^{\text {th }}$ level of transmission.

To go continuously from virulent to avirulent strains, we assume a trade-off between the different history traits of the virus (Anderson and May, 1982; Levin and Pimentel, 1981; May and Anderson, 1983). The mean duration of infection is a function of the transmission rate $D_{i}=D_{0}\left(\beta_{0} / \beta_{i}\right)^{\theta_{1}}$, where $D_{0}$ is the duration of the infection induced by strain $N_{V} . \theta_{l}$ represents how quickly the duration of the infection decreases with the transmission rate. The case mortality rate also depends on the transmission rate through $m_{i}=1-\left[1+\xi\left(\beta_{i} / \beta_{0}\right)^{\theta_{2}}\right]^{-1}$, where $\xi=\left(1-m_{0}\right)^{-1}-1, m_{0}$ is the case mortality of strain $N_{V}$ and $\theta_{2}$ describes how quickly the case mortality increases with the transmission rate. The rate of additional mortality $\alpha$ induced by the disease (which is here the virulence of the strain) and the recovery rate $\sigma$ are derived simply by the relations: 


$$
\begin{aligned}
& \alpha_{i}=\frac{m_{i}}{D_{i}}=\xi D_{0}^{-1} \beta_{0}^{-\theta_{1}} \frac{\beta_{i}^{\theta_{1}+\theta_{2}}}{\beta_{0}^{\theta_{2}}+\xi \beta_{i}^{\theta_{2}}} \\
& \sigma_{i}=\frac{1-m_{i}}{D_{i}}=D_{0}^{-1} \beta_{0}^{\theta_{2}-\theta_{1}} \frac{\beta_{i}^{\theta_{1}}}{\beta_{0}^{\theta_{2}}+\xi \beta_{i}^{\theta_{2}}}
\end{aligned}
$$

We choose $\theta_{1}$ and $\theta_{2}$ positive and so the virulence $\alpha$ is an increasing function of $\beta_{i}$. Hence we can also speak of more virulent strains for the strains that have a higher transmission rate. We assume that mutations may occur only during the transmission of the parasite with a probability $P_{V}$. To simplify, we assume that mutations from strain $i$ to strain $j$ are equally likely whatever $i$ and $j, i \neq j$. The probability that a strain $i$ mutates into a strain $j$ is then $\pi_{V}=P_{V} /\left(N_{V^{-}} 1\right)$.

Finally, we choose $\theta_{l}<1$, which allows generating a conflict between the local competitiveness of the strains, defined by the rank of their $R_{0}$ (see below), and their within host persistence expressed through $D_{i}$. As we will see later the most locally competitive strains (highest $R_{0}$ ) are the most virulent ones, whereas the most locally persistent strains are, especially for small subpopulations sizes, less virulent.

\section{Modelling the metapopulation structure. Rabbits are organized into well-defined social} groups that form a population (Cowan, 1987). Populations are connected together to form a higher structure level. We simplify this two levels structure by considering only one level of spatial organisation. We model the rabbit population as divided into $N_{S}$ subpopulations, which represent either the social groups or the populations, depending on which kind of level we consider the metapopulation to represent (a population or a set of connected populations, respectively). These subpopulations are set in a grid square of side length $\sqrt{N_{S}}$, each subpopulation being divided into susceptible, recovered and the $N_{V}$ infected compartments. 
The carrying capacity of each subpopulation is the same $(K)$ and thus the density-dependent mortality rate, $\mu_{0}+\delta N^{k}$, of the $k^{\text {th }}$ subpopulation depends only on its size $N^{k}$.

In the model, newborns are supposed to be susceptible. This is a simplification, since in fact rabbits younger than 2 months old have a partial resistance against RHDV (Ferreira et al., 2004; Morisse et al., 1991). Here we simplify the problem by assuming that the resistance of juveniles is perfect: they cannot get infected by any strain. Assuming that newborns are susceptible is then correct if births in the model correspond to resistance loss of young rabbits in the real system.

Juvenile rabbits may disperse when they become subadults (Kunkele and von Holst, 1996; Myers and Poole, 1961; Parer, 1982). This dispersion is taken into account because of its importance for the genetic structure of the population. It is possible that during a short period young rabbits acquire the infection and then disperse, transmitting the virus to their arrival subpopulation. However, as the results of the model would not change qualitatively if we neglect infectious dispersers, we limit the complexity of the model by separating the demographic and epidemiologic exchanges between subpopulations. In other words, we assume that dispersing individuals do not carry any virus with them. A natural way to achieve this is to assume that dispersers are born directly in their arrival subpopulation. To simplify we also assume that dispersion occurs only between neighbouring subpopulations. We call $\pi_{D}$ the probability of dispersal of a juvenile in each neighbouring subpopulation, which is constant whatever the position of the subpopulation (on the border or not). We call $P_{D}=8 \pi_{D}$, which corresponds, for subpopulations that are not on an edge of the square, to the total probability of dispersal. In the model, if the subpopulation is on the border then the total probability of dispersal becomes lower.

One infected individual from a subpopulation $k$ may infect individuals from different subpopulations $l$ at a rate that does not depend on $k$ and $l$. To simplify we assume that all 
foreign subpopulations are equally connected from the virus point of view. This can be a good approximation for example when subpopulations are social groups. In that case, rabbits from different groups meet principally in feeding areas and thus spatial distance between the different groups has little impact on the between group spread of the virus. Intersubpopulations transmission rate is assumed to be always $\rho$ times the intra-subpopulation one, which means that the most transmissible strains within a subpopulation are also the most transmissible between subpopulations.

The corresponding set of differential equations for subpopulation $k$ and strain $i\left(i=1 . . N_{V}\right)$ is:

$$
\begin{aligned}
& \frac{d S^{k}}{d t}=b\left[1-\Omega^{k} \pi_{D}\right] N^{k}+b \pi_{D} \sum_{l \in \eta^{k}} N^{l}-\left(\mu_{0}+\delta N^{k}\right) S^{k}-\left(\sum_{i=1}^{N_{V}} \Lambda_{i}^{k}\right) S^{k} \\
& \frac{d I_{i}^{k}}{d t}=\Lambda_{i}^{k} S^{k}-\left[\mu_{0}+\delta N^{k}+\alpha_{i}+\sigma_{i}\right] I_{i}^{k} \\
& \frac{d R^{k}}{d t}=\sum_{i=1}^{N_{V}} \sigma_{i} I_{i}^{k}-\left(\mu_{0}+\delta N^{k}\right) R^{k}
\end{aligned}
$$

where $S^{k}, I_{i}^{k}, R^{k}$ are the total numbers of individuals belonging to the $k^{\text {th }}$ subpopulation in the compartment $S, I_{i}, R$ respectively, and $N^{k}=S^{k}+\Sigma_{\mathrm{i}} I_{i}^{k}+R^{k} . \eta^{k}$ is the set of neighbours of the $k^{\text {th }}$ subpopulation, $\Omega^{k}$ the cardinal of $\eta^{k}$ (i.e. the number of neighbours of the $k^{\text {th }}$ subpopulation), and :

$$
\Lambda_{i}^{k}=\beta_{i}\left[1-\pi_{V}\left(N_{V}-1\right)\right]\left[I_{i}^{k}+\rho \sum_{l \neq k} I_{i}^{l}\right]+\sum_{j \neq i} \beta_{j} \pi_{V}\left[I_{j}^{k}+\rho \sum_{l \neq k} I_{j}^{l}\right]
$$

is the rate at which susceptible individuals from the subpopulation $k$ get infected by the strain $i$.

The stochastic version of the model, which takes demographic stochasticity into account, consists of a continuous time Markov chain in which transition rates are inferred 
from the deterministic model (see electronic supplementary materials). Since the global extinction of RHDV is not observed in the field, we assume that it is reintroduced through the immigration of an infected rabbit, e.g. from another metapopulation (not explicitly modelled here), at a rate $\varepsilon$. The strain carried by this infected rabbit as well as the subpopulation where it is introduced are chosen randomly with uniform laws. The rate at which an individual infected by the $i^{\text {th }}$ strain is introduced in the $k^{\text {th }}$ subpopulation is then $\varepsilon /\left(N_{S} N_{V}\right)$.

\section{c. Model with host genetic heterogeneity}

Now we assume two phenotypes for rabbits: secretors $(\boldsymbol{S E})$ and non-secretors $(\boldsymbol{s e})$. Each strain of RHDV is able to infect one and only one of the two phenotypes.

All compartments are divided into three sub-compartments: the non-secretors which are necessarily homozygote (se/se, se being recessive), the heterozygote secretors (SE/se) and the homozygote secretors $(\boldsymbol{S E} / \mathrm{SE})$.

Inactivating mutation on the gene $\boldsymbol{S E}$ may occur with a probability $\pi_{S E \rightarrow s e}$. The probability of the reverse mutation $\pi_{s e \rightarrow S E}$ is far lower. Indeed, the genotype $\boldsymbol{S E}$ represents the production of a specific $\alpha 1,2$ fucosyltransferase and random mutations of $\boldsymbol{S E}$ allele into se allele that inhibit this production are much more likely to occur than reverting mutations that allow recovery of a functional enzyme with similar catalytic properties (se allele into $\boldsymbol{S E}$ allele). In other words, it is far more likely that a mutation inactivates an activated gene than the contrary.

Here individuals are preferentialy infected by strains that are adapted to their genotype but a strain may mutate so as to infect the other phenotype and becomes then totally adapted to this new phenotype. The transmission rate between individuals of different phenotypes is $\rho_{P}$ times the transmission rate between two individuals of the same phenotype. More details about the genetic model are given into the appendix. 


\section{d. Parameter values}

Table 1 contains all the parameter values, which lead to values for the strain characteristics given in Table 2. The unit for time is the month. The birth rate is chosen to balance the annual natural mortality rate which is around $50 \%$ in the disease-free equilibrium (Cowan, 1987; Smith and Trout, 1994). The initial mortality rate $\mu_{0}$ is chosen very low compared to the birth rate, consistently with the invasive potential of rabbits. The probability $\left(P_{D}\right)$ of dispersal of newborns may vary strongly between populations (Richardson et al., 2002). Here we choose $50 \%$ as a basic value (Kunkele and von Holst, 1996; Richardson et al., 2002) but we will consider different values. For the basic parameters, the metapopulation is made up of on average 2450 rabbits equally distributed into 49 subpopulations $(K=50$ rabbits per subpopulation). The infection lasts 2 days on average and $95 \%$ of the rabbits infected by the virulent strain (strain $N_{V}$, where $N_{V}=10$ ) die from the infection.

Several parameters such as the transmission rate of all strains or the characteristics of intermediate strains remain poorly known. We study the effect of 5 parameters independently (i.e., anything else being equal): the carrying capacity of subpopulations $(K)$, which rules the effects of demographic stochasticity, the case mortality of the virulent strain $\left(m_{0}\right)$ (which affects the case mortality of the other strains, see above), the level of connectivity between subpopulations $(\rho)$, the local transmission rate of the virulent strain $\left(\beta_{0}\right)$ and the host birth rate (b). However, the effects of some parameters overlap. For example, increasing the subpopulation carrying capacity $(K)$ will obviously decrease the importance of stochastic effects within the subpopulation, which is the phenomenon we are interested in, but will also increase the local transmission due to the mass action choice for the incidence function. The effect of increasing the local transmission of the virus is already studied with $\beta_{0}$. In order to isolate the effect of stochasticity associated with variable host subpopulation size, we choose 
a transmission rate $\beta$ inversely proportional to $K$ (see Table 1 and electronic supplementary materials for more details) to keep the within host subpopulation transmission of the virus constant. The same problem appears with the local transmission rate of the virulent strain $\beta_{0}$. The rate of contamination of one susceptible individual by an infected one belonging to a different subpopulation is $\rho \beta_{i}$. To maintain a constant transmission rate between subpopulations, we choose the connectivity rate $\rho$ inversely proportional to the transmission rate. More precisely, as the transmission of the virus also depends on the size of the subpopulation (due to the mass action choice), we choose $\rho$ inversely proportional to $\beta K$ (see Table 1 and electronic supplementary materials for more details).

The stochastic model is treated through numerical simulations beginning with an initial state where the number of susceptible individuals is $K$ in each subpopulation and one individual infected by strain $N_{V}$ is released in the down-left corner subpopulation. Initially, each metapopulation is made up of $K$ susceptible individuals. In the genetic model the initial gene frequency is 50-50 (in panmictic proportions). One $\boldsymbol{S E}$ individual carrying the virulent strain is introduced into the left corner subpopulation. For each set of parameters in the stochastic version of the models, we consider 10 replicates of the process. This allows us to estimate the variability of the system, from which we derive estimations of the means and confidence intervals for the variables we aim to estimate.

\section{2) $\underline{\text { Results }}$}

a. The deterministic approach

The introduction of RHDV in the fully susceptible host metapopulation is followed by a very rapid epidemic (so rapid here that it can hardly be seen in Figure 2a). The number of infected individuals then rapidly reaches a steady state (here after two years). The most virulent strain $\left(N_{v}\right)$ is always selected. Other strains suffer an exponential decay until 0 (they are not 
represented on Figure 2a). The selection of the most virulent strain is due to a classical result of epidemiology based on the selection of the strains with the highest basic reproductive number $R_{0}$. The $R_{0}$ of the strain $i$ is given by:

$$
R_{0}\left(V_{i}\right)=\frac{\beta_{i} K\left[1+\rho\left(N_{S}-1\right)\right]}{b+\alpha_{i}+\sigma_{i}}=\left[1+\rho\left(N_{S}-1\right)\right] \frac{\beta_{i} K}{b+D_{0}^{-1}\left(\frac{\beta_{i}}{\beta_{0}}\right)^{\theta_{1}}}
$$

Here, as $\theta_{1}<1, R_{0}$ is an increasing function of the transmission rate and is thus maximum for the most virulent strain (strain $N v$, see also Table 2). Note that this is true independently of the number of subpopulations and their carrying capacity. In other words, the structure of the host population does not affect the evolution of RHDV. For any value of $N_{S}$, subpopulations rapidly synchronize, so the results obtained in the simplest case $\left(N_{S}=1\right.$, see Figure 2$)$ are representative of the results obtained for larger values of $N_{S}$ (not shown).

The presence of the virus has a strong and rapid impact on the rabbit population size (Figure 2b). Host polymorphism has a very low impact on both the number of infected rabbits (see Figure $2 \mathrm{a}$ ) and the size of the rabbit population (see Figure $2 \mathrm{~b}$ ).

At the equilibrium, genetic diversity is maintained when the virus is present (Figure 2c). This is due to the frequency-dependent fitness of individuals. For example, when the group $\boldsymbol{S E}$ is larger than the group se, strains adapted to $\boldsymbol{S E}$ spread more efficiently, infecting a greater proportion of hosts $\boldsymbol{S E}$. The fitness of an individual $\boldsymbol{S E}$ is then reduced, which decreases the number of $\boldsymbol{S E}$. Conversely, in a disease-free population, polymorphism is not maintained: secretors go extinct (see Figure 2c) due to asymetrical mutations.

Surprisingly, the time required to reach equilibrium between secretors and non-secretors increases with the transmission rate (Figure $2 \mathrm{~d}$ ). This is because when the transmission rate increases rabbits have less chance of escaping the infection. When the transmission rate is high, belonging to a less abundant phenotypic group only slightly decreases the probability of 
getting infected, which is high anyway. The consequence of this is that the density dependent pressure tends to decrease with the transmission rate.

At the equilibrium, the number of infected individuals per subpopulation is largely below 1 (see Figure 2a), and at this stage stochastic fluctuations cannot be neglected anymore. In reality, the number of infected individuals should fall to 0 , which corresponds to the extinction of the virus. The predictions of the deterministic model thus have to be treated with caution, and a stochastic version of the model is needed for more realism.

\section{b. The stochastic approach}

i. Evolution of virulence in absence of host genetic diversity

The stochastic model shows much more contrasted results than the deterministic one. Since local extinction can occur, the long-term persistence of the strains (at the scale of the metapopulation) depends on their ability to colonize other subpopulations in addition to their persistence at the subpopulation level. Figure 3 illustrates a typical situation where a low virulent strain is selected. Higher virulent strains may invade disease-free subpopulations, or even replace strain 3 in some infected subpopulations. But as soon as the more virulent strains have gone extinct from one subpopulation, this disease-free subpopulation can be recolonized by strain 3 , which has been maintained in many other subpopulations. On average, the balance between extinction and recolonization is in favour of strain 3, which is finally selected over the entire metapopulation.

To understand how the structure of the host metapopulation and the characteristics of the pathogen affect the optimal viral strategy, we explore a wide range of parameter values.

To do so we define the "mean level of virulence" of RHDV by $\frac{\sum_{i, k} i \times I_{i}^{k}}{\sum_{i, k} I_{i}^{k}}$. Even if this "mean 
virulence level" by itself has no biological meaning, it is directly related to important pathogen history traits. In particular, the mean value of the logarithm of the virus transmission rate is linearly related to the mean virulence. It thus indicates the mean rank of virulence relatively to the range of virulence displayed by the set of strains considered.

The effect of the host subpopulation size on the mean virulence of RHDV (Figure 4a) illustrates how different subpopulation structures may favour different strains. Basically, the success of a strain depends on how many subpopulations it is able to colonize before going extinct - either through natural extinction or strain replacement - from its original subpopulation. In very small subpopulations, all strains are poorly persistent, so the virus is transmitted to other subpopulations mostly during the epidemic that occurs right after the introduction of the strain. Highly virulent strains induce a huge epidemic and are easily transmitted to other subpopulations, which explains the high mean virulence levels at very low subpopulation sizes (see Figure 4a). As the subpopulation size increases, less virulent strains become able to persist in an endemic state over long periods after the end of the initial epidemic. The mean virulence of RHDV decreases. However, with increased subpopulation size these strains persist longer. The very long persistence of low virulent strains provides no more advantage since they are replaced long before going naturally extinct. The mean virulence of RHDV increases (see Figure 4a).

A direct consequence of this is that the mean level of virulence of RHDV increases with the probability of survival from infection (Figure $4 b$ ). When all strains are more lethal the competition is even more severe since less virulent strains reduce less the number of rabbits in the subpopulations and hence spread in larger host subpopulations, which increases their persistence advantage over more virulent strains.

A higher connectivity between subpopulations favours less locally (and thus less within host) persistent strains (Figure 4c) because persisting within a subpopulation is not so 
advantageous when strains both highly virulent and transmissible can colonize rapidly other subpopulations and locally outcompete more locally persistent but less transmissible strains. As a result, the mean virulence of RHDV increases with the connectivity between subpopulations.

The mean level of virulence increases with the birth rate (Figure 4d) for the same reason as for the host subpopulation size: a higher birth rate leads to faster replacement of susceptible rabbits for RHDV, permitting a longer persistence of each strain. More virulent strains, which are more transmissible, are also selected and can persist longer compared to previously, pinpointing the high degree of persistence that can be achieved when the host population is rapidly replaced by newborns.

Finally, we explore the impact of the local transmission rate, keeping the transmission rate between subpopulations $\left(\beta_{0 .} \rho\left(N_{S^{-}} 1\right)\right)$ constant by changing in consequence the value of the connectivity between subpopulations $(\rho)$. We observe a decrease in the mean virulence of RHDV with increasing local transmission rate. This is because highly transmissible strains consume the potentially susceptible pool too quickly when local transmission rates are higher. Moreover, the stability of low virulent strains is improved since a high local transmissibility reduces the susceptible pool in their subpopulation, limiting the risk of invasion by a more virulent and competitive strain. In contrast, when the local transmission rate of a strain is low, a large part of the host subpopulation remains susceptible and more virulent strains can easily spread in the subpopulation.

One can note that rabbit abundance varies inversely with the mean virulence, since the impact of the virus on the host population is more severe when induced by more virulent strains. This remark is valid for every graph in this figure except $4 b$, where the selection of more virulent strains is caused by an increase in the survival of infected rabbits, which accounts for the reduced impact on host abundance. 


\section{ii. Impact of host genetic diversity on RHDV virulence}

The impact of genetic diversity on the virulence of RHDV is a direct consequence of the results seen above. The genetic diversity splits the host population into two subgroups: the secretors and the non-secretors. From the virus point of view, all strains are only able to infect one phenotype, so all happens as if the part of the rabbit population with the other phenotype did not exist. This has two consequences. First, the rabbit subpopulation size in which the virus spreads is lower. This implies a higher demographic stochasticity in the host population (studied in Figure 4a) and also a lower rate of contamination of susceptible individuals (due to the mass action law; the effect of the transmission rate is studied in Figure 4e). Second, due to the variability in the local frequency of each allele, some subpopulations are free from one of the two phenotypes. The number of subpopulations in which a strain can spread is thus lower, which reduces the frequency of contamination between subpopulations (studied in Figure 4c). These points affect the mean virulence in different ways.

In fact, in most situations, the host polymorphism decreases the mean virulence of RHDV (Figure 5). As an exception, no such impact is observed for small subpopulation sizes (Figure 5a), low survival to virulent strain (Figure 5b), low birth rates (Figure 5d) and low local transmission rate (Figure 5e). For small subpopulations sizes, the reason is certainly a low level of persistence of the polymorphism (see below). The virus does not really spread in a heterogeneous host population. This also explains the absence of an obvious decrease in RHDV virulence with host genetic diversity in the case where RHDV is highly lethal (see Figure 5b).

Figure 5d shows some interesting results. For three different points, we observe very small confident intervals. For two of these points the effect of host genetic diversity is close to 
0 (let us call them points A and B, point A corresponding to the lowest birth rate). For the third one it is close to -1 (say point C). When looking in more detail, one can see that in this region of the birth rate, strains rarely coexist in equivalent proportion. Most of the time one strain excludes the other, although this strain may differ from one replicate to the next. A very small confident interval means that for both the genetic and the non-genetic models one strain (that can differ between the two models) will have a high probability of excluding the others (one can also see that on Figure 4d). In this region, virulence of RHDV increases by steps (see Figure 4d). The rapid shift from point B to point $C$ means that the threshold value of the birth rate for which the mean level of virulence of RHDV increases by 1 is lower for the nongenetic model than for the genetic one.

The only case where the host genetic polymorphism leads to an increase in the mean virulence of RHDV is that of low transmission rates. As seen previously, a low transmission rate of the virus favours more transmissible strains by making infected subpopulations easier to colonize. The host polymorphism increases this effect by decreasing the local transmission of the virus, favouring more virulent strains.

\section{iii. Maintenance of host genetic diversity}

According to the deterministic model, the presence of the microparasite should favour the maintenance of the host genetic diversity. Results from the stochastic model are once again more complex. In such models, global extinction of genes may occur just by chance. Due to the very low probability of mutation from $\boldsymbol{s} \boldsymbol{e}$ to $\boldsymbol{S E}$, in the long term the rabbit population will certainly converge toward the extinction of the $\boldsymbol{S E}$ gene. The question is then how long it takes and will the virus help to maintain the genetic diversity over a longer period?

The global persistence of RHDV is not the only relevant question here. Rabbit populations are variable in many aspects. In particular, the number of subpopulations within 
the metapopulation may vary greatly between populations. Subpopulations loose and exchange genes, which leads to a kind of equilibrium in the proportion of subpopulations carrying each gene. This proportion is globally preserved when the number of subpopulations varies, but for a small number of subpopulations fluctuations in this proportion may lead to the global extinction of a gene. Clearly genes that are represented in a low proportion of subpopulations will tend to go extinct first. For this reason we also choose the frequency of local extinction of each gene as an indicator of the stability of the host genetic polymorphism.

Another argument for this choice is that global extinction of genes is, for most values of the parameters tested here, very rare. Estimating the time before extinction of a gene would require very long simulations. Instead, we prefer to look at the state of the system after long periods, here basically between 150 and 200 years after the introduction of the virus. On an ecological scale, it is already a very long period of time. It is sufficient for stabilizing the evolution of the virus virulence but not the evolution of the genetic diversity.

First we look at the effect of the probability of juvenile dispersion on the evolution of genetic diversity (Figure 6a, b). For basic values of the parameters $\left(P_{D}=0.5\right)$ almost no local gene extinction is expected (see Figure 6a, b). Unsurprisingly, the frequency of gene extinction decreases with the genetic connectivity between subpopulations (i.e. the juvenile dispersion). The results clearly show the combined effects of the asymmetric mutation rate and of the dominant character of the $\boldsymbol{S E}$ gene: the $\boldsymbol{S E}$ gene is more likely to go locally extinct (see Figure 6a) than the se gene (see Figure 6b).

Surprisingly, RHDV is found to have a negative impact on the persistence of the gene $\boldsymbol{S E}$ (see Figure 6a) and a positive impact on the persistence of the gene se (see Figure 6b). The fact that $\boldsymbol{S E}$ is more likely to go extinct than se implies that RHDV is detrimental to the rabbit polymorphism. To look at this in more detail and understand how RHDV affects the probability of persistence of each gene, we define a new basic probability of juvenile 
dispersion. Indeed, with $50 \%$ of juvenile dispersion we expect only rare local gene extinction. It is not convenient to study the impact of the parameters.

We now define as new basic value of the probability of juvenile dispersion $P_{D}=0.02$. We first study how the system evolves with time (Figure 6c-f). Results clearly show that 200 years is not sufficient to reach equilibrium. The frequency of each phenotype (Figure 6c) and of local extinction of each gene (Figure 6d) still evolve after 1000 years. Global extinction of the $\boldsymbol{S E}$ gene is not observed before 400 years, and even after 1000 years the SE gene has gone globally extinct in only $20 \%$ ( $40 \%$ in the case without virus) of the replicates (Figure $6 \mathrm{e}$ ). The $\boldsymbol{s e}$ gene has never gone globally extinct in any of the 50 replicates.

This trend toward a higher global extinction probability of the $\boldsymbol{S E}$ gene without RHDV is confirmed by looking at the evolution of the frequency of $\boldsymbol{S E}$ local extinction with time (Figure 6f). In the first 500 years RHDV accelerates the extinction of the $\boldsymbol{S E}$ gene. This is not surprising since initially all subpopulations have a perfect genetic balance. RHDV kills many rabbits and has a large destabilizing effect on this balance. However, as the time runs, the system reaches an equilibrium where RHDV strains are long term persistent and induce a moderate mortality. The virus pressure is regular and density-dependent, which has a stabilizing effect on the genetic diversity. Finally, after 1000 years, RHDV significantly decreases the frequency of local extinction of the $\boldsymbol{S E}$ gene (see Figure 6f).

Unsurprisingly, the frequency of local $\boldsymbol{S E}$ extinction decreases with the host subpopulation size (Figure 7a). This is simply due to the fact that larger subpopulations are less prone to genetic drift. Interestingly, the frequency of local $\boldsymbol{S E}$ extinction decreases with the probability of survival to RHDV (Figure 7b). In other words more lethal strains are more detrimental to the maintenance of the genetic diversity. This is because by killing rabbits RHDV destabilizes the genetic balance within subpopulations, increasing the rate of local 
gene extinction. Of course, one has to remember that this occurs only after 200 years.

Looking at a larger time scale might reverse the trend.

Other parameters are found to have no obvious impact on the frequency of $\boldsymbol{S E}$ extinction (Figure 7c-e). This does not mean that they have no impact at all, since they affect the mean virulence of RHDV (see Figure 4c-e), but rather that particular mechanisms balance the effect of these changes in the mean virulence. Indeed, the selection of high virulent strains is often associated with a higher proportion of infected subpopulations. One explanation could be that higher infestation of the metapopulation permits a high frequency of multiple infected subpopulations and hence favours the strains that are good local competitors. Another explanation could be that more virulent strains are more transmissible. The worst situation for the persistence of a gene is when a phenotypic group is infected and not the other. This situation tends to become rarer as more subpopulations are infected. This explains why despite affecting the mean virulence of RHDV, the connectivity between subpopulations (Figure 7c), the birth rate (Figure 7d) and the local transmission rate (Figure 7e) do not really affect the frequency of $\boldsymbol{S E}$ local extinction.

\section{3) Discussion}

\section{Maintenance of the genetic diversity}

In the present paper we developed two modelling approaches. In the deterministic model, the high mortality induced by the virus limits the host population at a low level where the host polymorphism persists, whereas in the absence of virus, the asymmetric mutation rate drives the $\boldsymbol{S E}$ allele towards extinction. This is due to the frequency-dependent fitness of the host, which decreases with the relative size of the genetic group he belongs to (Agrawal and Lively, 2003). However, the maintenance of the host genetic polymorphism was clearly dependent on the long-term persistence of the virus, for the study of which the deterministic model does not 
seem appropriate, since a very low number of infected hosts was maintained at the equilibrium. More realistically we should observe extinctions of the virus. For that reason we turn to the stochastic version of the model to integrate virus extinction and reintroduction events by considering demographic fluctuations.

The stochastic model allows going one step further into this analysis. The virus has a stabilizing impact on the host polymorphism, which increases the chance of genetic diversity maintenance in the long term. However, on an ecological time scale, the virus has been found to have a negative impact on the persistence of the genetic diversity. Without the virus, there is a balance between the host phenotypes which surely but slowly drives the secretor allele toward extinction. In contrast, the virus destabilizes the balance by killing preferentially one phenotype or the other, which may lead to rapid local allele extinction.

If the model cannot predict the minimum host population size required to maintain the host polymorphism, it is certainly more appropriate to determine the kind of parameters that may influence the maintenance of the host polymorphism. Apart from the subpopulation size which, unsurprisingly, positively affects the maintenance of the host polymorphism, the only parameter which has here a significant effect is the case mortality of the virus. The more the virus kills the host the worse it is for the short-term persistence of the polymorphism.

\section{Effect of spatial and genetic host diversity on strain selection}

Deterministic and stochastic versions of the same model largely differ in their predictions concerning the evolution of the virus. With the parameters we chose, the deterministic model predicts that the most virulent strain should always be selected whatever the characteristics of the host metapopulation, owing to its highest $R_{0}$. In contrast, the stochastic model predicts that the evolution of the virus virulence is much more complex. In particular, despite the strong competitive advantage of highly virulent strains, the stochastic model shows that the latter are 
unadapted to small subpopulations because they go extinct rapidly, thereby limiting their chances of colonizing other subpopulations. However, more virulent strains are better local competitors and are favoured when exchanges of parasites between subpopulations are more frequent. The trade-off between the persistence of the virus within subpopulations and its capacity to colonize other subpopulations leads to the selection of intermediate virulent strains, but depends on several factors. When a factor favours the persistence of all strains, the parasite transmission rate between subpopulations or reduces strains' resistance against invaders, selection favours more transmissible but also more virulent strains. In particular, high birth rate, low virus induced mortality, high connectivity between subpopulations, low local transmission rate and very large and very small host subpopulations favour highly virulent strains. On the contrary, genetic diversity, by decreasing the size of the host population available for each strain, generally selects for less virulent strains.

The model we developed is based on the assumption of a trade-off between the three main parasite traits: the transmission rate, the duration of infectiousness and the case mortality induced by the parasite. In reality, these three traits are not necessarily linked, or at least less explicitly. It could thus be important to isolate their respective effects. Two characteristics are particularly important in the competition between parasites: the parasite transmission rate and the total time during which the parasite can be transmitted (directly or indirectly through e.g., vectors or infected ground). The parasite will evolve to maximize these two traits if they are independent, and if not to a compromise between these traits, favouring more persistent strains in small and poorly connected subpopulations (see above). The last trait is the capacity of the parasite to reduce the host population size (here through the case mortality), which is important because it reduces the chances of persistence of all strains and hence favours more locally persistent ones (it has the same effect as a reduction in the subpopulation carrying capacity). 


\section{$\underline{\text { Individual vs population-based modelling approaches }}$}

Keeling (2000) already explored parasite evolution within a metapopulation framework. He used an approach centred on the subpopulation, a method commonly used to represent metapopulations. However, as has been previously pointed out (Keeling, 2002), these models can be interpreted as approximations of models based on the individual. It is thus important to estimate the consequences of this choice. Focusing here on the individual, we confirmed the qualitative results found by Keeling (2000), i.e. that the capacity of a pathogen to persist locally is a very important trait for its evolution. We confirm the general idea that in a more stochastic host demography and/or in less connected subpopulations, more persistent strains tend to be selected.

However, our model allows to go deeper into the analysis of the problem. It allows determining how the characteristics of the strains interplay with subpopulation characteristics. For example, strains that invest in persistence characteristics (i.e. infected individuals transmit them over long periods) have no persistence advantage in very small subpopulations.

The persistence of a strain within a host subpopulation or the capacity of a more virulent strain to invade an already infected subpopulation are complex functions that depend virtually on all the demographic and epidemiologic parameters of the model. In particular, there is a feed-back between the parasite evolution and the demography of the host: the host demography influences the evolution of the parasite, which in turn influences the host population size, favouring more or less virulent strains.

Many parameters act in a complex and sometimes contradictory manner on both of these functions and their impact cannot be effectively determined without considering realistic models. Our approach allows testing explicit demographic (birth rate, host subpopulation size, 
level of connectivity between subpopulations), epidemiologic (transmission rate) and genetic (of both hosts and parasites) characteristics of the host-parasite interaction.

\section{Validity of the model}

Another important difference with the model proposed by Keeling (2000) is that in our case the host population is not made up of infinity of subpopulations (an intrinsic constraint of the deterministic character of Keeling's model). With infinity of subpopulations, and with a connectivity of one between subpopulations, we would have found the selection of strains with the highest basic reproductive number (results not shown). This was not the case here mainly because the entire metapopulation was not large enough for the persistence of the most virulent strain. Moreover, we observe little variability in our results, which means that in most replicates the same strain predominated in an endemic state (results not shown). What is interesting in this situation is that the predominating strain can be invaded by more virulent strains. More virulent strains can spread, but never long enough to compromise the persistence of the predominant strain. This is typically the kind of situation where the evolution of the virus depends on the size of the entire metapopulation and/or on the frequency of reintroduction of each strain (and also on the circulation of the virus at the upper scale and the degree of isolation of the metapopulation).

Does that mean that the model is insufficient? No, the model is adapted to study the evolution of the virus in the metapopulation, provided that external reintroductions are infrequent enough to have no weight in the evolution of the virus. In any other situation one should look at an upper scale, i.e. a set of connected metapopulations.

Among the questions that have not been treated here is the effect of the host metapopulation size. One could suppose that highly virulent strains can be selected, but only in very large and highly connected metapopulations. Further simulations of the model (not 
presented here) confirm this idea: in larger metapopulations more virulent strains can be selected provided there is a high connectivity between subpopulations. However, the global patterns observed here remain true for larger metapopulations. It could also be attractive to test much smaller metapopulation sizes to estimate the minimum number of rabbits required to maintain the host polymorphism. Unfortunately this does not provide useful results. In small metapopulations no virus is long term globally persistent. The metapopulation is often disease-free. After a virus reintroduction, one host phenotypic group is infected and reduced. The persistence of host genes thus depends on the frequency of external reintroductions, the virulence of the reintroduced strain and the phenotype infected. All these factors cannot be modelled properly, simply because they depend on the circulation of the virus on a larger scale. Only looking at larger scales can allow to answer all these questions.

Finally, it is important to note that the paper is based on simplifying assumptions. For example, we ignored the fact that, although juveniles are resistant to the most severe forms of the disease, they can still get infected and transmit the virus to other rabbits. This may have important implications for the evolution of virus. For example, highly virulent strains can become more persistent if they spread only in juvenile rabbits and so be selected in the long term. The main difficulty is that we do not have enough information on this phenomenon so that some critical questions cannot be answered: how efficient is the transmission of the virus by resistant juveniles in the field? How are the strain characteristics affected when the infection occurs in juveniles?

Here we focused on general mechanisms illustrating how the competition between strains may resolve when there is a conflict between invasion and persistence. Even if juveniles might alter the characteristics of the different strains, RHDV is likely to face this conflict, so that, on a qualitative point of view, the results of the model remain informative. 


\section{Application to the rabbit-RHDV interaction}

The model is based on simplifying assumptions and the fact that some important parameter values are unknown prevents us from making reliable quantitative predictions. However, the model can be compared with qualitative observations and help to understand some important features of the rabbit-RHDV interaction. One striking observation is that, more than twenty years after the emergence of RHDV in China, both virulent and avirulent strains are still found in the field. This is surprising since the classical theory of parasite evolution states that when two strains of the same parasite compete, then the strain with the highest basic reproductive number excludes the other strain. Why has this not yet happened for RHDV?

One possible explanation is that the basic reproductive number of the avirulent strains varies between rabbit populations. White et al. (2001) have argued that avirulent strains of RHDV should induce very long lasting diseases to balance their poor level of transmission. They showed that in that case the basic reproductive number of avirulent strains depends on the life expectancy of rabbits, which varies between populations. So each strain can be locally selected in some areas but not in others and the diffusion of the virus between populations may explain their coexistence.

Our paper suggests another plausible and not exclusive explanation. The differential success between virulent and avirulent strains depends on rabbit population characteristics (e.g., size, connectivity). One can postulate that virulent strains are better adapted in some regions and are diffused from these regions to populations in which avirulent strains are better adapted.

Another reason why virulent strains of RHDV managed to persist until now could be that they could have evolved alternative persistence strategies. For example, recent evidence support the idea that RHDV could be long-term persistent in rabbits surviving acute infections 
(Gall and Schirrmeier, 2006). So virulent strains could persist in recovered rabbits until the renewal of the rabbit susceptible pool.

Another important property of RHDV is that until now no strain of intermediate virulence has been isolated in the field. This is in contradiction with our results (selection of intermediate virulent strains). This can be due to a physiological reason that makes RHDV either benign or highly lethal. But intermediate virulent strains may also exist and not be detected because: i) as argued previously, there is an observational bias in favour of highly virulent strains that induce spectacular demographic crashes in rabbit populations or ii) some other selection pressures select for extreme virulent strains only and finally leads to the low prevalence of intermediate virulent ones.

\section{$\underline{\text { Conclusion and implications }}$}

These results may explain observations from human-norovirus induced gastroenteritis. The capacity of a norovirus to infect a host also depends on the secretor phenotype of the host as shown by studies on volunteers (Hutson et al., 2005; Lindesmith et al., 2003). It has been demonstrated that some strains are able to infect only secretors while others are expected to infect non-secretors only (Le Pendu et al., 2006; Tan and Jiang, 2005). In parallel, field studies on authentic outbreaks conducted in Sweden revealed that all the strains recorded infected secretors only, leading the authors to wonder if non-secretors were resistant to all noroviruses (Thorven et al., 2005). Here we propose another scenario. In most countries, including Sweden, populations are made up of around 80\% of secretors (Mourant, 1983). According to our results, strains infecting secretors should be more transmissible but also less persistent, because they infect a larger group. If noroviruses' transmission rate increases with their induced morbidity and if the duration of the infectiousness decreases with morbidity, 
then strains infecting non secretors could be difficult to record simply because they induce no or little morbidity.

All of these results may have important consequences for the study of the evolution of pathogens. It is widely admitted that natural selection favours the highest $R_{0}$ strains in homogeneous host populations (Anderson and May, 1979; May and Anderson, 1983). In fact, strategies that improve the local persistence of the parasite may also be favoured in spatial host populations (see e.g. Haraguchi and Sasaki, 2000). In our model, when populations are made up of small and interconnected groups, the parasite also has to adapt to the size of the group by being able to persist long enough to be efficiently transmitted to other groups. Long infectious period with low levels of both transmissibility and virulence can be one such strategy. Alternative mechanisms of extension of the pathogen infectivity period beyond host infection are frequently observed in the field, as exemplified by the parvovirus infecting cats (Berthier et al., 2000) or the hantavirus infecting voles (Kallio et al., 2006; Sauvage et al., 2003) that can remain infectious in the ground. Myxoma virus, another virus infecting rabbits, provides another interesting example. The virus is able to persist over long periods in the ground (Joubert et al., 1974) or in infected vectors (Andrewes et al., 1956; Chapple and Lewis, 1965) and to spontaneously reactivate in recovered hosts several months after recovery (Marlier et al., 2000; Williams et al., 1972). This suggests that myxoma virus is well adapted to the spatial organisation of rabbits into small social groups.

\section{Acknowledgements:}

We thank the FNC and ANR SEST programme "Pathocénoses et émergence des maladies transmissibles: un concept unificateur mis à l'épreuve sur des pathologies exemplaires". We 
also thank one anonymous referee for his useful comments. The funders had no role in study design, data collection and analysis, decision to publish, or preparation of the manuscript.

\section{$\underline{\text { Author contributions: }}$}

DF built the model, performed the numerical simulations and wrote the paper. JLP is a specialist of the question of the rabbit polymorphism regarding RHDV. He discussed the model assumptions so that the model fits as well as possible the experimental system. MG helped in the model development, discussed the model assumptions. She was involved in the writing of the paper. JSG and SM are specialists of rabbits in the field. They discussed the model assumptions and helped determining the model parameters. DP is the supervisor of this work. She was also involved in the writing of the paper. All authors participated in the discussion of the model results.

All the authors declare that they have no conflict of interest that could potentially influence their work.

\section{Appendix: mathematical formulation of the genetic model}

For $X$ representing $S, I_{i}, R$ or $N$ we call $X_{S e}{ }^{k}, X_{S E / s e}{ }^{k}$ and $X_{S E}{ }^{k}$ the total number of individuals of the $k^{\text {th }}$ subpopulation belonging to the compartment $X$ that are respectively non-secretors, heterozygote secretors and homozygote secretors. Note that ${I_{i, S E}}^{k}$ and $I_{i, s e}{ }^{k}$ do not represent individuals infected by the same strain, the first one is adapted to secretors and the second one to non secretors, whereas $I_{i, S E}{ }^{k}$ and $I_{i, S E / s e}{ }^{k}$ represent individuals infected by the same strain. To simplify, we call strains $\boldsymbol{S E}$ (respectively $\boldsymbol{s} \boldsymbol{e}$ ) the strains adapted to the phenotype $\boldsymbol{S E}$ (respectively $\boldsymbol{s e}$ ).

The frequency of the gene $\boldsymbol{S E}$ in the $k^{\text {th }}$ subpopulation is then: 
$f_{S E}^{k}=\left[N_{S E}{ }^{k}+N_{S E / s e}{ }^{k} / 2\right] /\left[N_{S E}{ }^{k}+N_{S E / s e}{ }^{k}+N_{s e}{ }^{k}\right]$

To simplify, we assume a panmictic population and thus the proportions of young born with a given genotype in the subpopulation $k$ are given by:

$$
\begin{aligned}
& F_{S E / S E}{ }^{k}=\left[f_{S E}{ }^{k}\left(1-\pi_{S E \rightarrow s e}\right)+f_{s e}{ }^{k} \pi_{S e \rightarrow S E}\right]^{2} \\
& F_{S E / s e}{ }^{k}=2\left[f_{S E}{ }^{k}\left(1-\pi_{S E \rightarrow s e}\right)+f_{s e}{ }^{k} \pi_{s e \rightarrow S E}\right]\left[f_{S E}{ }^{k} \pi_{S E \rightarrow s e}+f_{s e}{ }^{k}\left(1-\pi_{s e \rightarrow S E}\right)\right] \\
& F_{\text {se/se }}{ }^{k}=\left[f_{S E}{ }^{k} \pi_{S E \rightarrow s e}+f_{s e}{ }^{k}\left(1-\pi_{s e \rightarrow S E}\right)\right]^{2}
\end{aligned}
$$

We can then establish the differential equations of the system. The exponent $k$ is used for the $k^{\text {th }}$ subpopulation $\left(k=1 . . N_{S}\right)$, the indexes $i$ for the $i^{\text {th }}$ level of transmission rate $\left(i=1 . . N_{V}\right)$ and $G$ for the genotype of the individual $(G=S E / S E, S E / s e$ or $\boldsymbol{s e} / \mathbf{s e})$ :

$$
\begin{aligned}
& \frac{d S_{G}^{k}}{d t}=b F_{G}^{k}\left[1-\Omega^{k} \pi_{D}\right] N^{k}+b \pi_{D} \sum_{l \in \eta^{k}} F_{G}^{l} N^{l}-\left(\mu_{0}+\delta N^{k}\right) S_{G}^{k}-\left(\sum_{i=1}^{N_{V}} \Lambda_{i, G}^{k}\right) S_{G}^{k} \\
& \frac{d I_{i, G}^{k}}{d t}=\Lambda_{i, G}^{k} S_{G}^{k}-\left[\mu_{0}+\delta N^{k}+\alpha_{i}+\sigma_{i}\right] I_{i, G}^{k} \\
& \frac{d R_{G}^{k}}{d t}=\sum_{i=1}^{N_{V}} \sigma_{i} I_{i, G}^{k}-\left(\mu_{0}+\delta N^{k}\right) R_{G}^{k}
\end{aligned}
$$

where

$$
\begin{aligned}
& \Lambda_{i, S E / S E}^{k}=\Lambda_{i, S E / s e}^{k}=\lambda_{i, S E / S E}^{k}+\lambda_{i, S E / s e}^{k}+\rho_{P} \lambda_{i, s e / s e}^{k} \\
& \Lambda_{i, s e / s e}^{k}=\rho_{P} \lambda_{i, S E / S E}^{k}+\rho_{P} \lambda_{i, S E / s e}^{k}+\lambda_{i, s e / s e}^{k}
\end{aligned}
$$

and

$$
\lambda_{i, G}^{k}=\beta_{i}\left[1-\pi_{V}\left(N_{V}-1\right)\right]\left[I_{i, G}^{k}+\rho \sum_{l \neq k} I_{i, G}^{l}\right]+\sum_{j \neq i} \beta_{j} \pi_{V}\left[I_{j, G}^{k}+\rho \sum_{l \neq k} I_{j, G}^{l}\right]
$$

is the rate at which susceptible individuals from the subpopulation $k$ get infected with the strain $i$ by individuals with a genotype $G$.

As previously, the stochastic version of the genetic model consists of a continuous time Markov chain in which transition rates are given by the rates of the deterministic model (see 
electronic supplementary notes). As in the non-genetic model, the release of an infected individual in the metapopulation at a rate $\varepsilon$ prevents the definitive extinction of RHDV. Subpopulation and strain for reintroduction are chosen according to the same uniform laws as above. The genotype of the individual is chosen randomly according to the frequency of the three genotypes in the metapopulation. This simple hypothesis ensures that an extinct genotype cannot be reintroduced by immigration from outside the metapopulation.

\section{$\underline{\text { References: }}$}

Agrawal, A.F., Lively, C.M., 2003. Modelling infection as a two-step process combining gene-for-gene and matching-allele genetics. Proc. R. Soc. Lond. B. 270, 323-334.

Alitzer, S., Dobson, A., Hosseini, P., Hudson, P., Pascual, M., Rohani, P., 2006. Seasonality and the dynamics of infectious diseases. Ecol. Lett. 9, 467-484.

Anderson, R.M., May, R.M., 1979. Population biology of infectious diseases. Nat. Med. 280, $361-367$.

Anderson, R.M., May, R.M., 1981. The population dynamics of microparasites and their invertebrate hosts. Philos. Trans. R. Soc. Lond. Ser. B 291, 451-524.

Anderson, R.M., May, R.M., 1982. Coevolution of hosts and parasites. Parasitology 85, 411 426.

Andrewes, C.H., Muirhead-Thomson, R.C., Stevenson, J.P., 1956. Laboratory studies of Anopheles atroparvus in relation to myxomatosis. J. Hyg. 54, 478-486.

Bell, G., Maynard Smith, J., 1987. Short-term selection for recombination among mutually antagonistic species. Nature 328, 66-68.

Berthier, K., Langlais, M., Auger, P., 2000. Dynamics of a feline virus with two transmission modes with exponentially growing host population. Proc. R. Soc. Lond. B. 267, 20492056. 
Boots, M., Sasaki, A., 1999. "small world" and the evolution of virulence: infection occurs locally and at distance. Proc. R. Soc. Lond. B. 266, 1933-1938.

Boots, M., Mealor, M., 2007. Local interactions select for lower pathogen infectivity. Science $315,1284-1286$.

Boots, M., Hudson, P.J., Sasaki, A., 2004. Large shifts in pathogen virulence relate to host population structure. Science 303, 842 - 844 .

Calvete, C., 2006. Modeling the effect of population dynamics on the impact of rabbit hemorrhagic disease. Conserv. Biol. 20, 1232-1241.

Capucci, L., Fusi, P., Lavazza, A., Pacciarini, M.L., Rossi, C., 1996. Detection and preliminary characterization of a new rabbit calicivirus related to rabbit hemorrhagic disease virus but nonpathogenic. J. Virol. 70, 8614-8623.

Chapple, P.J., Lewis, N.D., 1965. Myxomatosis and the rabbit flea. Nature 207.

Chasey, D., Lucas, M.H., Westcott, D.G., Sharp, G., Kitching, A., Hughes, S.K., 1995. Development of a diagnostic approach to the identification of rabbit haemorrhagic disease. Vet. Rec. 137, 158-160.

Claessen, D., de Roos, A.M., 1995. Evolution of virulence in a host-pathogen system with local pathogen transmission. Oikos 74, 401-413.

Clarke, B., 1976. The ecological genetics of host-parasite relationships. In: Taylor, A. E. R. and Muller, R., (Eds.), Genetic aspects of host-parasite relationships. Blackwell, London, pp. 87-103.

Cooke, B.D., Robinson, A.J., Merchant, J.C., Nardin, A., Capucci, L., 2000. Use of ELISAs in field studies of rabbit haemorrhagic disease (RHD) in Australia. Epid. Infect. 124, 563-576.

Cowan, D.P., 1987. Aspects of the social organisation of the European wild rabbit (Oryctolagus cuniculus). Ethology 75, 197-210. 
Dieckmann, U., Metz, J.A.J., Sabelis, M.W., Sigmund, K. (Eds.), 2002. Adaptive dynamics of infectious diseases: in pursuit of virulence management. Cambridge University Press, Cambridge, UK.

Dwyer, G., Levin, S.A., Buttel, L., 1990. A simulation model of the population dynamics and evolution of myxomatosis. Ecol. Monogr. 60, 423-447.

Fenner, F., 1953. Changes in the mortality rate due to myxomatosis in the Australian wild rabbit. Nature 172, 228.

Ferreira, P.G., Costa-e-Silva, A., Monteiro, E., Oliveira, M.J.R., Aguas, A.P., 2004. Transient decrease in blood heterophils and sustained liver damage caused by a calicivirus infection of young rabbits that are naturally resistant to rabbit haemorrhagic disease. Res. Vet. Sci. 76, 83-94.

Gall, A., Schirrmeier, H., 2006. Persistence of Rabbit Haemorrhagic Disease Virus genome in vaccinated rabbits after experimental infection. J. Vet. Med. B 53, 358-362.

Hamilton, W.D., 1980. Sex versus non-sex versus parasite. Oikos 35, 282-290.

Hamilton, W.D., Axelrod, R., Tanese, R., 1990. Sexual reproduction as an adaptation to resist parasites. Proc. Natl. Acad. Sci. USA 87, 3566-3573.

Haraguchi, Y., Sasaki, A., 2000. Evolution of parasite virulence and transmission rate in a spatially structured population. J. theor. Biol. 203, 85-96.

Howard, R.S., Lively, C.M., 1994. Parasitism, mutation accumulation and the maintenance of sex. Nature $367,554-557$.

Hughes, W.O.H., Boomsma, J.J., 2004. Genetic diversity and disease resistance in leaf-cutting ant societies. Evolution 58, 1251-1260.

Hutson, A.M., Airaud, F., Le Pendu, J., Estes, M.K., Atmar, R.L., 2005. Norwalk virus infection associates with secretor status genotyped from sera. J. Med. Virol. 77, 116120. 
Joubert, L., Massis, S., Prave, M., 1974. Conservation interépizootique prolongée du virus myxomateux. Med. Mal. Infect. 4, 349-354.

Kallio, E.R., Klingström, J., Gustafsson, E., Manni, T., Vaheri, A., Henttonen, H., Vapalahti, O., Lundkvist, A., 2006. Prolonged survival of Puumala hantavirus outside the host: evidence for indirect transmission via the environment. J. Gen. Virol. 87, 2127-2134.

Keeling, M.J., 2000. Evolutionary trade-offs at two time-scales: competition versus persistence. Proc. R. Soc. Lond. B. 267, 385-391.

Keeling, M.J., 2002. Using individual-based simulations to test the Levins metapopulation paradigm. J. Anim. Ecol. 71, 270-279.

Kunkele, J., von Holst, D., 1996. Natal dispersal in the European wild rabbit. Anim. Behav. $51,1047-1059$.

Le Gall-Reculé, G., Zwingelstein, F., Laurent, S., De Boisseson, C., Portejoie, Y., Rasschaert, D., 2003. Phylogenetic analysis of Rabbit haemorrhagic disease virus in France between 1993 and 2000, and the characterisation of RHDV antigenic variants. Arch. Virol. 148, 65-81.

Le Pendu, J., Ruvöen-Clouet, N., Kindberg, E., Svensson, L., 2006. Mendelian resistance to human norovirus infections. Semin. Immunol. 18, 375-386.

Levin, S.A., Pimentel, D., 1981. Selection for intermediate rates of increase in parasite-host systems. Am. Nat. 117, 308-315.

Lindesmith, L., Moe, C.L., Marionneau, S., Ruvoën-clouet, N., Jiang, X., Lindblad, L., Stewart, P.A., Le Pendu , J., Baric, R.S., 2003. Human susceptibility and resistance to Norwalk virus infection. Nat. Med. 9, 548-553.

Liu, S.J., Xue, H.P., Pu, B.Q., Qian, N.H., 1984. A new viral disease of rabbits. Anim. Husband. Vet. Med. 16, 253-255. 
Marchandeau, S., Boucraut-Baralon, C., 1999. Epidémiologie de la myxomatose et des caliciviroses apparentées à la VHD au sein d'une population sauvage de lapins de garenne. Gibier Faune Sauvage, Game Wildl. 16, 65-80.

Marchandeau, S., Ricci, J.-C., Chantal, J., 1998. Taux de prévalence sérologique du virus de la maladie virale hémorragique (VHD) du lapin de garenne (Oryctolagus cuniculus) et de ses formes apparentées au sein de différentes populations sauvages en France. Mammalia 62, 95-103.

Marionneau, S., Cailleau-Thomas, A., Rocher, J., Le Moullac-Vaidye, B., Ruvoën-clouet, N., Clément, M., Le Pendu, J., 2001. ABH and Lewis histo-blood group antigens, a model for the meaning of oligosaccharide diversity in the face of a changing world. Biochimie 83, 565-573.

Marlier, D., Mainil, J., Sulon, J., Beckers, J.F., Linden, A., Vindevogel, H., 2000. Study of the virulence of five strains of amyxomatous myxoma virus in crossbred New Zealand white/Californian conventional rabbits, with evidence of long-term testicular infection in recovered animals. J. Comp. Pathol. 122, 101-113.

May, R.M., Anderson, R.M., 1983. Epidemiology and genetics in the coevolution of parasites and hosts. Proc. Roy. Soc. B. 219, 281-313.

Morisse, J.-P., Le Gall, G., Boilletot, E., 1991. Hepatitis of viral origin in Leporidae: introduction and aetiological hypotheses. Revue Scientifique et Technique - Office International des Epizooties 10, 283-295.

Moss, S.R., Turner, S.L., Trout, R.C., White, P.J., Hudson, P.J., Desai, A., Armesto, M., Forrester, N.L., Gould, E.A., 2002. Molecular epidemiology of rabbit haemorrhagic disease virus. J. Gen. Virol. 83, 2461-2467.

Mourant, A.E., 1983. Blood relations: blood groups and anthropology. Oxford University Press, Oxford. 
Myers, K., Poole, W.E., 1961. A study of the biology of the wild rabbit, Oryctolagus cuniculus (L.), in confined populations II. The effects of season and population increase on behaviour. CSIRO Wildl. Res. 6, 1-41.

O'Brien, S.J., Evermann, J.F., 1988. Interactive influence of infectious-disease and genetic diversity in natural populations. Trends Ecol. Evol. 3, 254-259.

Parer, I., 1982. Dispersal of the wild rabbit, Oryctolagus cuniculus, at Urana in New South Wales. Austr. Wildl. Res. 9, 427-441.

Pearman, P.B., Garner, T.W.J., 2005. Susceptibility of Italian agile frog populations to an emerging strain of Ranavirus parallels population genetic diversity. Ecol. Letters 8, 401-408.

Rand, D.A., Keeling, M.J., Wilson, H.B., 1995. Invasion, stability and evolution to criticality in spatially extended, artificial host-pathogen ecology. Proc. R. Soc. Lond. B. 259, 5563.

Richardson, B.J., Hayes, R.A., Wheeler, S.H., Yardin, M.R., 2002. Social structures, genetic structures and dispersal strategies in Australian rabbit (Oryctolagus cuniculus) populations. Behav. Ecol. Sociobiol. 51, 113-121.

Rodak, L., Smid, B., Valicek, L., Vesely, T., Stepanek, J., Hampl, J., Jurak, E., 1990. Enzyme-linked immunosorbent assay of antibodies to rabbit haemorrhagic disease virus and determination of its major structural proteins. J. Gen. Virol. 71, 1075-1080.

Ruvoën-Clouet, N., Ganière, J.P., André-Fontaine, G., Blanchard, D., Le Pendu, J., 2000.

Binding of rabbit hemorrhagic disease virus to antigens of the ABH histo-blood group family. J. Virol. 74, 11950-11954.

Sanson, R.L., Brooks, H.V., Horner, G.W., 2000. An epidemiological study of the spread of rabbit haemorrhagic disease virus amongst previously non-exposed rabbit populations in the North Island of New Zealand. N. Z. Vet. J. 48, 105-110. 
Sauvage, F., Langlais, M., Yoccoz, N.G., Pontier, D., 2003. Modelling hantavirus in fluctuating populations of bank voles: the role of indirect transmission on virus persistence. J. Anim. Ecol. 72, 1-13.

Siemens, D., Roy, B., 2005. Tests for parasite-mediated frequency-dependent selection in natural populations of an asexual plant species. Evol. Ecol. 19, 321-338.

Smith, G.C., Trout, R.C., 1994. Using Leslie matrices to determine wild rabbit population growth and the potential for control. J. Appl. Ecol. 15, 223-230.

Tan, M., Jiang, X., 2005. Norovirus and its histo-blood group antigen receptors: an answer to a historical puzzle. Trends Microbiol. 13, 285-293.

Thorven, M., Grahn, A., Hedlund, K.-O., Johansson, H., Wahlfrid, C., Larson, G., Svensson, L., 2005. A homozygous nonsense mutation $(428 \mathrm{G}>\mathrm{A})$ in the human FUT2 gene provides resistance to symptomatic norovirus (GGII) infections. J. Virol. 79, 1535115355.

Trout, R.C., Chasey, D., Sharp, G., 1997. Seroepidemiology of rabbit haemorrhagic disease (RHD) in wild rabbits (Oryctolagus cuniculus) in the United Kingdom. J. Zool. 243, 846-853.

White, P.J., Norman, R.A., Trout, R.C., Gould, E.A., Hudson, P.J., 2001. The emergence of rabbit haemorrhagic disease virus: will a non-pathogenic strain protect the UK? Phil. Trans. R. Soc. Lond. B 356, 1087-1095.

Williams, R.T., Dunsmore, J.D., Parer, I., 1972. Evidence for the existence of latent myxoma virus in rabbits (Oryctolaqus cuniculus (L.)). Nature 238, 99-101. 
Table 1: basic values of the parameters and their relationships. The time unit is the month.

\begin{tabular}{|c|c|c|c|}
\hline Parameter & Symbol & $\begin{array}{l}\text { Relationship to } \\
\text { other parameters }\end{array}$ & $\begin{array}{l}\text { Basic } \\
\text { value }\end{array}$ \\
\hline Number of subpopulations & $N_{S}$ & & 49 \\
\hline Subpopulation carrying capacity & $K$ & $K=2450 / \mathrm{N}_{\mathrm{S}}$ & 50 \\
\hline Birth rate & $b$ & & $6 \times 10^{-2}$ \\
\hline Initial mortality rate & $\mu_{0}$ & $\mu_{0}=\mathrm{b} / 10$ & $6 \times 10^{-3}$ \\
\hline Density-dependent mortality rate & $\delta$ & & $1.1 \times 10^{-3}$ \\
\hline Transmission rate of the virulent strain & $\beta_{0}$ & $\beta_{0}=2000 / K$ & 40 \\
\hline Inter-subpopulations relative transmission rate & $\rho$ & $\rho=1 / \beta_{0} K$ & $10^{-4}$ \\
\hline $\begin{array}{l}\text { Decrease of the duration of the infection rate with the } \\
\text { transmission rate }\end{array}$ & $\theta$ & & 0.83 \\
\hline $\begin{array}{l}\text { Increase of the case mortality with the transmission } \\
\text { rate }\end{array}$ & $\theta_{2}$ & & 0.83 \\
\hline Mean duration of the infection by the virulent strain & $D_{0}$ & & $1 / 15$ \\
\hline Case mortality of the infection by the virulent strain & $m_{0}$ & & 0.95 \\
\hline Number of strains infecting each phenotype & $N_{V}$ & & 10 \\
\hline Probability of change in virus virulence & $P_{V}$ & & $10^{-3}$ \\
\hline Probability of juvenile dispersal & $P_{D}$ & & 0.5 \\
\hline Probability of mutation from $S E$ to $s e$ & $\pi_{S E \rightarrow s e}$ & & $10^{-3}$ \\
\hline Probability of mutation from se to $S E$ & $\pi_{s e \rightarrow S E}$ & & $10^{-6}$ \\
\hline Infection by an individual having a different phenotype & $\rho_{P}$ & & $10^{-3}$ \\
\hline Reintroduction rate (only stochastic models) & $\varepsilon$ & & $1 / 12$ \\
\hline
\end{tabular}


Table 2: characteristics of the 10 strains for the basic value of the parameters

\begin{tabular}{lllll}
\hline Strain & $\begin{array}{l}\text { Transmission } \\
\text { rate }\end{array}$ & $\begin{array}{l}\text { Duration of the } \\
\text { infectious period }\end{array}$ & $\begin{array}{l}\text { Case mortality } \\
(\mathbf{\%})\end{array}$ & $\boldsymbol{R}_{\boldsymbol{0}}$ \\
\hline 1 & 0.01 & 66.7 & 1.86 & 6.70 \\
2 & 0.025 & 30.9 & 3.93 & 13.7 \\
3 & 0.063 & 14.4 & 8.10 & 24.5 \\
4 & 0.16 & 6.67 & 16.0 & 37.9 \\
5 & 0.40 & 3.09 & 29.0 & 52.2 \\
6 & 1.00 & 1.44 & 46.9 & 66.4 \\
7 & 2.53 & 0.667 & 65.5 & 80.9 \\
8 & 6.34 & 0.309 & 80.4 & 96.3 \\
9 & 15.9 & 0.144 & 89.8 & 113.4 \\
\hline 10 & 40 & 0.0667 & 95.0 & 132.8 \\
\hline
\end{tabular}




\section{$\underline{\text { Figure captions: }}$}

Figure 1: Flow diagram of the non genetic model in the $k$-th subpopulation with infection by infectious rabbit carrying the strain $i$. Doted lines represent the fact that infectious individuals play a role in the contamination but are not changed by it. Grey cells represent the compartments and white ones the rates of transition between compartments. Bold lines represent the flows with the highest rates: transmission between two individuals of the same subpopulation and the susceptible individual infected by the same strain as the infectious one. However, the contamination may be due to an infectious individual from another subpopulation $l$ and/or the strain may mute during the infection process and become strain $j$. The dark filled circle represents the possibility of mutation after infection. Demographic events (i.e. births and deaths), dispersion and external reintroduction of the virus are not represented here.

Figure 2: Results from the deterministic model for basic parameter values, except $N_{S}=1$ subpopulation. (a) Number of individuals infected by strain $N_{v}$ in the genetic (solid lines) and non-genetic (dashed lines) models; (b) number of rabbits with time in the genetic (solid lines) and non-genetic (dashed lines) models; (c) proportion of secretors with time under the virus pressure (solid line) or without virus (dashed line) and (d) proportion of individuals with the secretor phenotype with time under the virus pressure for basic values of the same parameters as in (c) (bold line) and for a smaller local transmission rate of the virulent strain $\left(\beta_{0}=10\right.$, thin line). 
Figure 3: Number of rabbits per subpopulation infected by (a) strain 3; (b) strain 4; (c) strain 5 ; and (d) any other strain (i.e. strains 1, 2, 6, 7, 8, 9 and 10). Results are shown for one replicate, using the basic value of the parameters.

Figure 4: Mean virulence of RHDV (points, left axis) with 95\% CI (vertical bars) and abundance index of rabbit, defined by the mean number of rabbits per subpopulation divided by the carrying capacity of the subpopulation (grey, right axis, the width of the line indicate the $95 \% \mathrm{CI}$ ) in the non-genetic model, calculated over the last 50 years of a 200 years simulation, according to: (a) the carrying capacity $(K)$ of subpopulations (the number of rabbits in the entire metapopulation being constant); (b) the probability that infected individuals recover from the most virulent strain $\left(1-m_{0}\right)$; (c) the level of connection between subpopulations $(\rho) ;(\mathrm{d})$ the host birth rate $(b)$ and $(\mathrm{e})$ the local transmission rate $\left(\beta_{0}\right)$

Figure 5: Impact of the host genetic diversity on the evolution of RHDV. Mean difference (with 95\% CI) in the virulence of RHDV between the genetic model and the non-genetic model (all other parameters being equals), calculated over the last 50 years of a 200 years simulation. (a) According to the carrying capacity $(K)$ of subpopulations; (b) the probability that infected individuals recover from the most virulent strain $\left(1-m_{0}\right)$; (c) the level of connection between subpopulations $(\rho)$; (d) the host birth rate $(b)$ and (e) the local transmission rate $\left(\beta_{0}\right)$. On each subplot, the grey area represents the region where host polymorphism decreases the mean virulence of RHDV (i.e. negative difference).

Figure 6: Persistence of the genetic diversity in presence or absence of RHDV. Mean proportion of subpopulations (with $95 \% \mathrm{CI}$ ) where the $\boldsymbol{S E}$ gene (a) or the se gene (b) is extinct, calculated over the last 50 years of a 200 years simulation, according to the 
probability of dispersion of juveniles $\left(P_{D}\right)$ with (solid lines) or without (doted lines) RHDV (using 10 replicates for each point). (c-f) Evolution of the host polymorphism with time, with $P_{D}=0.02$. (c) Proportion of non-secretor in the entire metapopulation (using 50 replicates); (d) proportion of subpopulations where $\boldsymbol{S E}$ (black) and $\boldsymbol{s} \boldsymbol{e}$ (grey) genes are extinct; (e) proportion of simulations where the $\boldsymbol{S E}$ gene is extinct from the entire metapopulation with (bold line) and without (thin line) RHDV. The se gene never goes globally extinct in any of the 50 replicates. (f) Effect of RHDV on the genetic diversity, characterized by the difference in the frequency of local extinction of the gene $\boldsymbol{S E}$ with time between the case with and the case without RHDV. Non-secretors invade more rapidly with RHDV but in the long term the virus slows down the decay of the number of secretors. In $(c, d, f)$, the width of the line represents the $95 \% \mathrm{CI}$ of the estimated variable.

Figure 7: Impact of the model parameters on the evolution of genetic diversity, represented by the mean proportion (with $95 \% \mathrm{CI}$ ) of subpopulations where the $\boldsymbol{S E}$ gene is extinct with (solid lines) or without (doted lines) RHDV, calculated over the last 50 years of a 200 years simulation. (a) According to the carrying capacity $(K)$ of subpopulations; (b) the probability that infected individuals recover from the most virulent strain $\left(1-m_{0}\right)$; (c) the level of connection between subpopulations $(\rho)$; (d) the host birth rate $(b)$ and (e) the local transmission rate $\left(\beta_{0}\right)$. In (b), (c) and (e) the model without RHDV is the same for all points and so is the frequency of local $\boldsymbol{S E}$ extinction. 


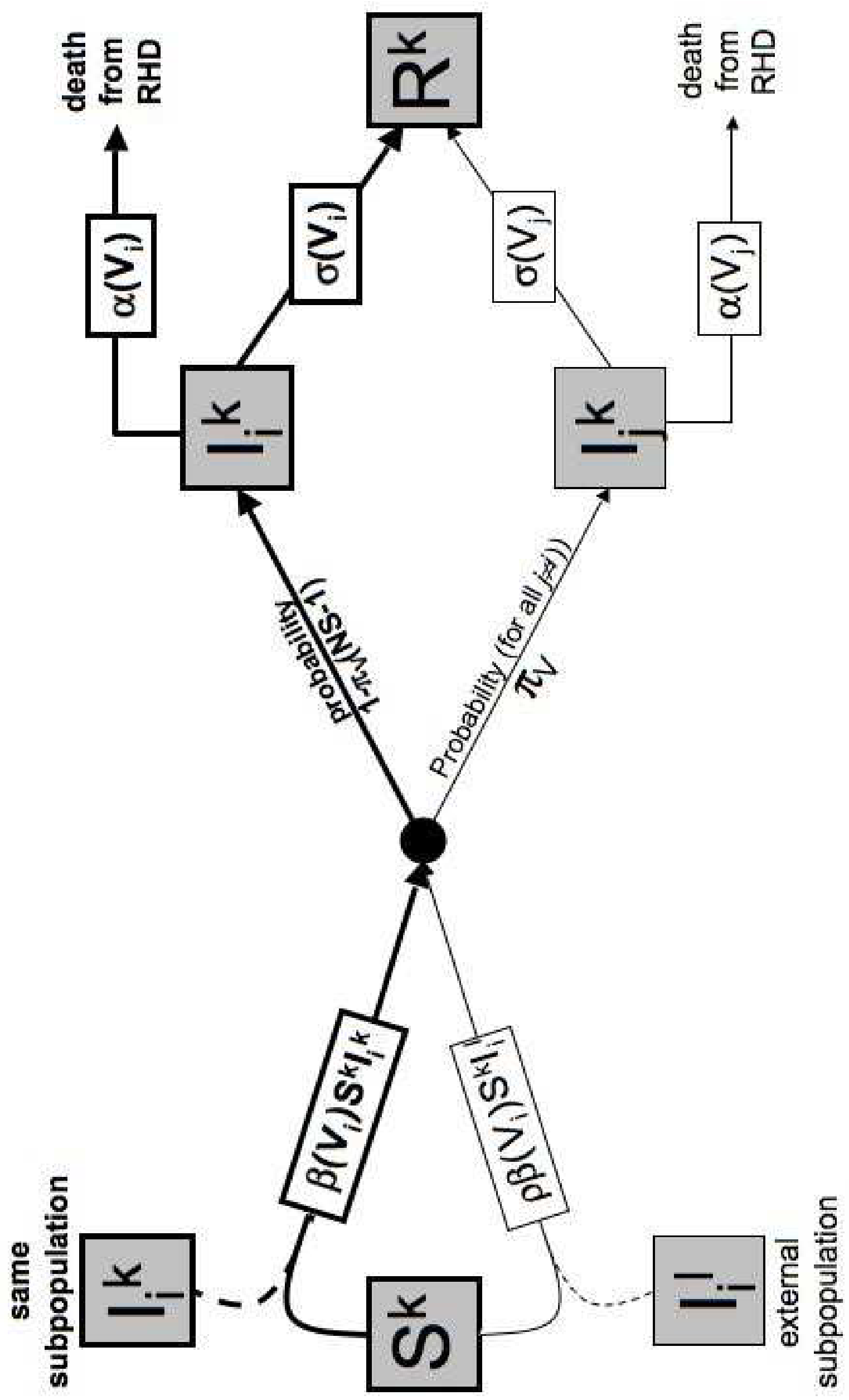



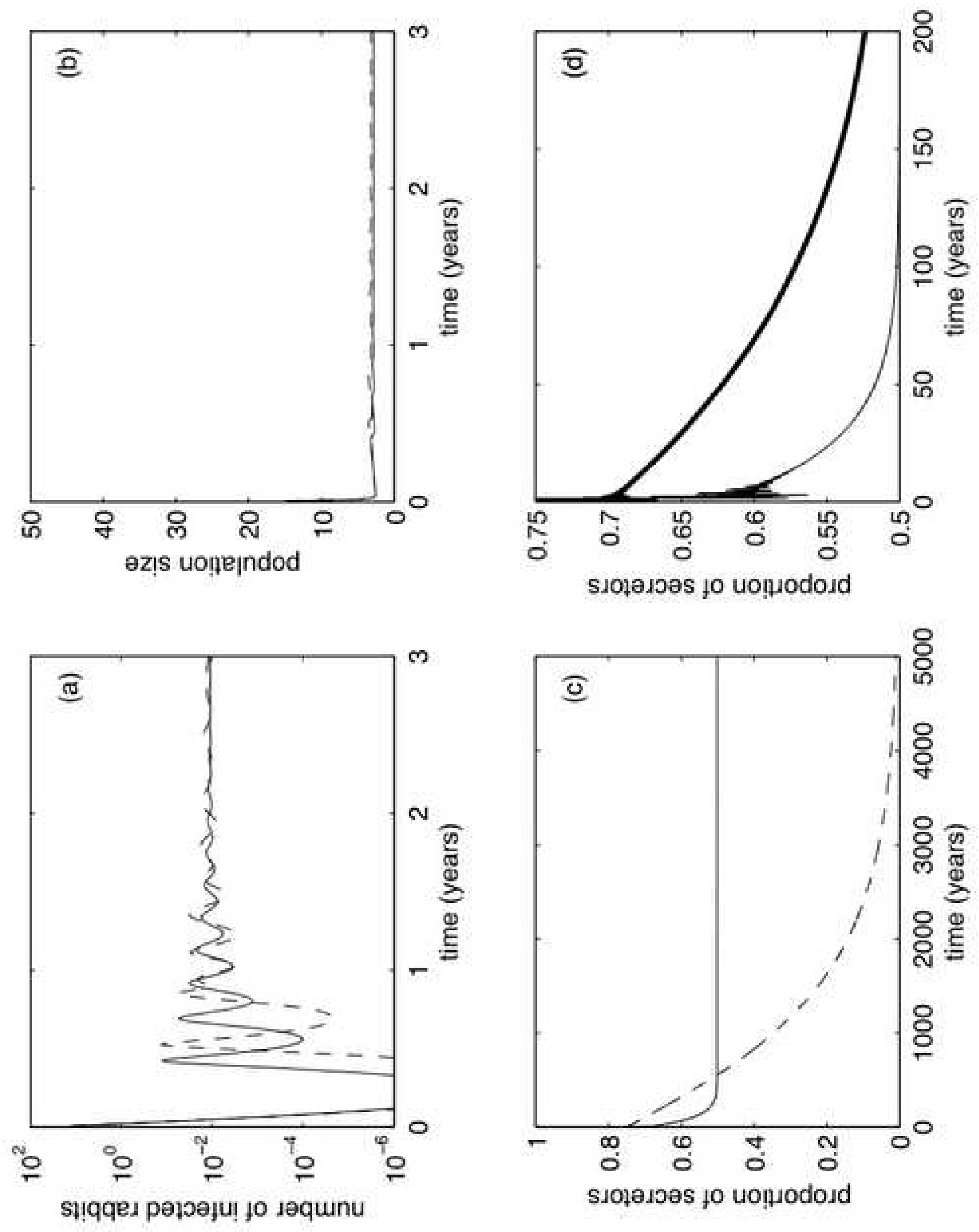


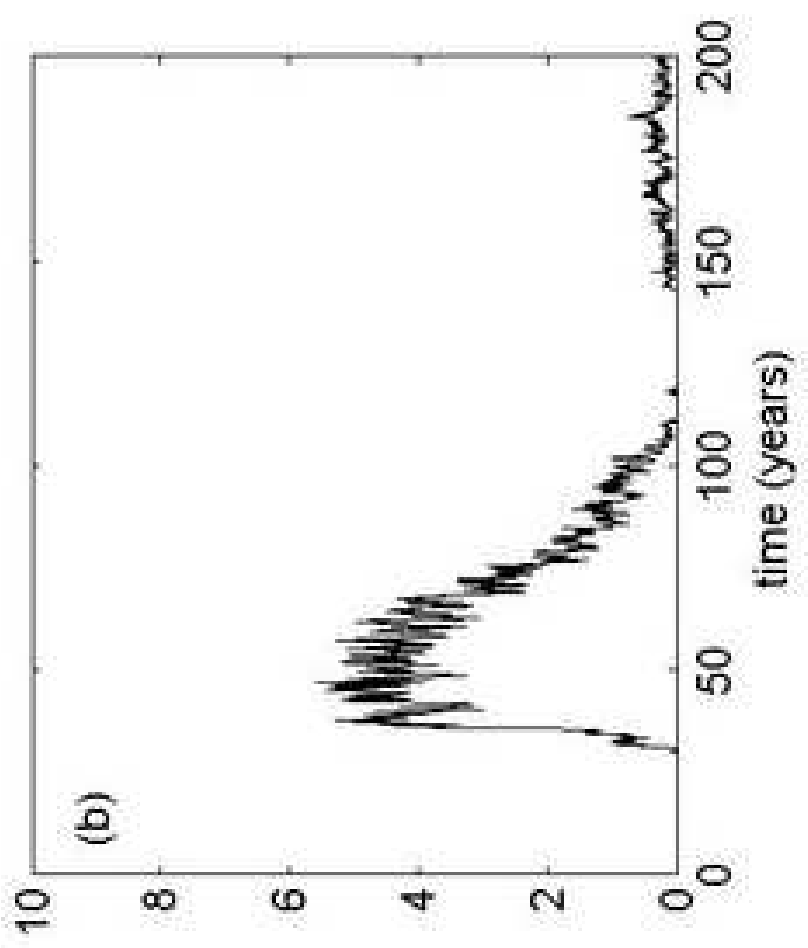

† Uịens

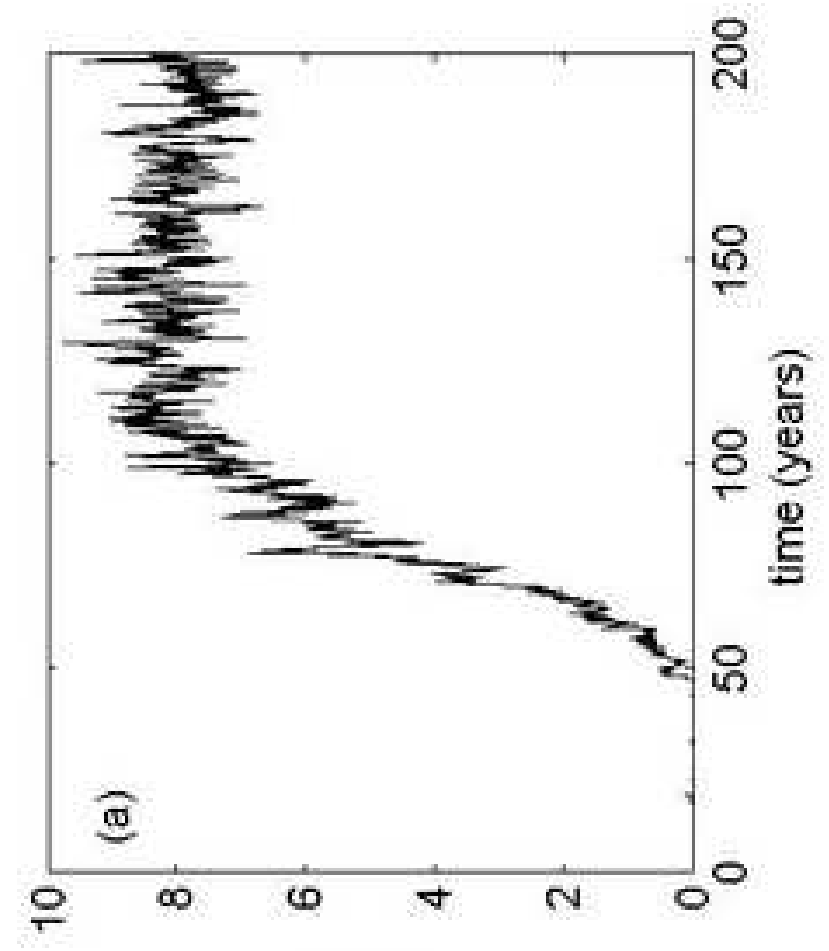

$\varepsilon$ uịedls
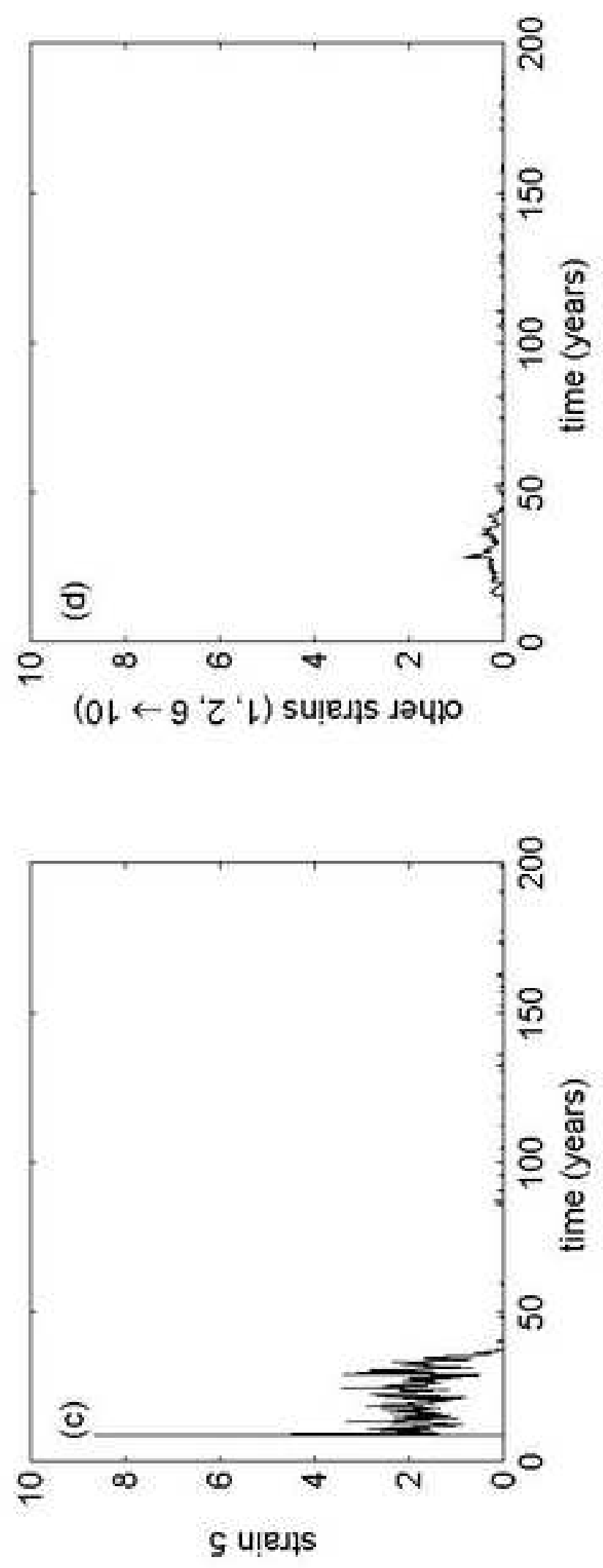

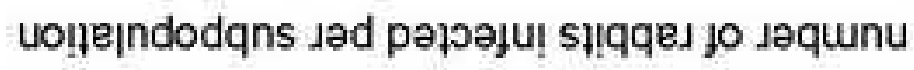




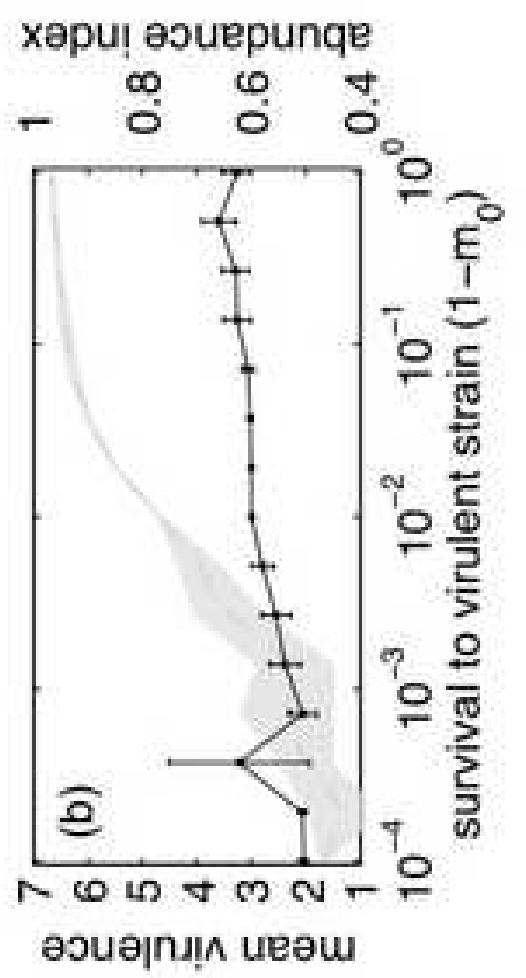

xapul әэuepunqe

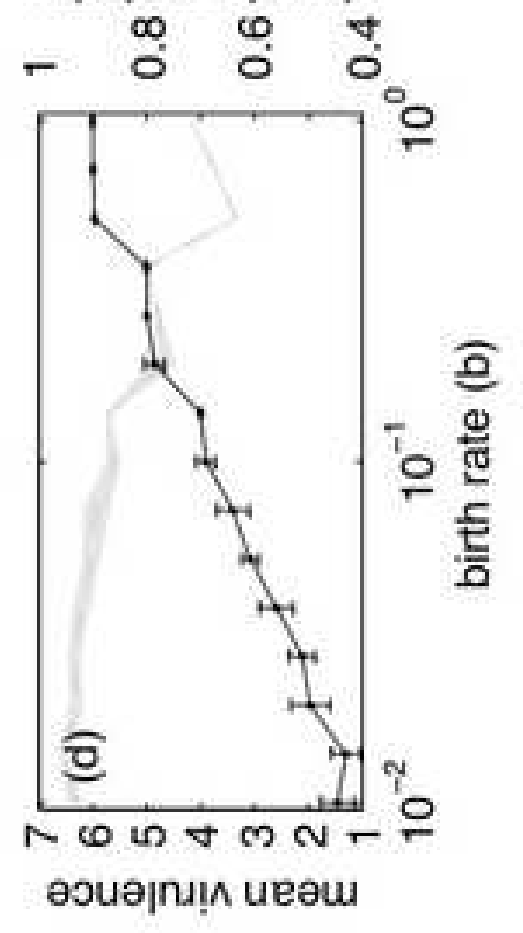

xapul әouepunqe

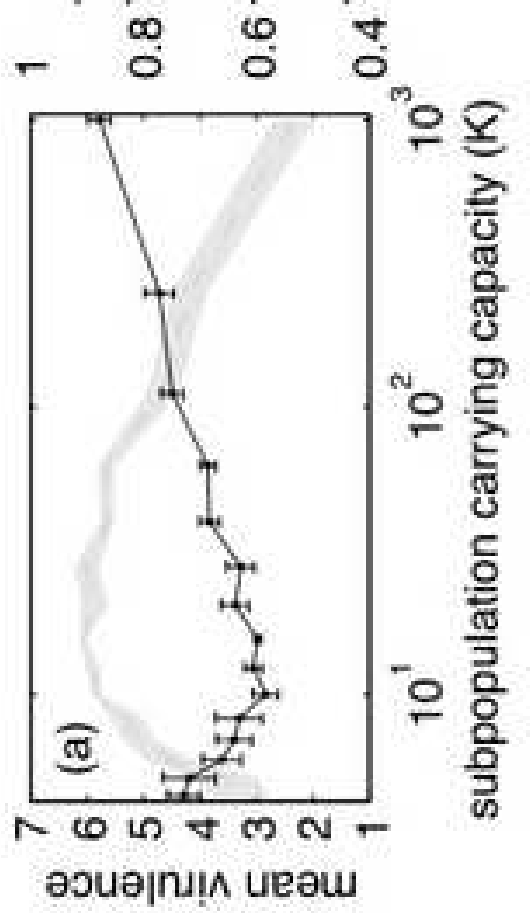

xepu! әouepunqe

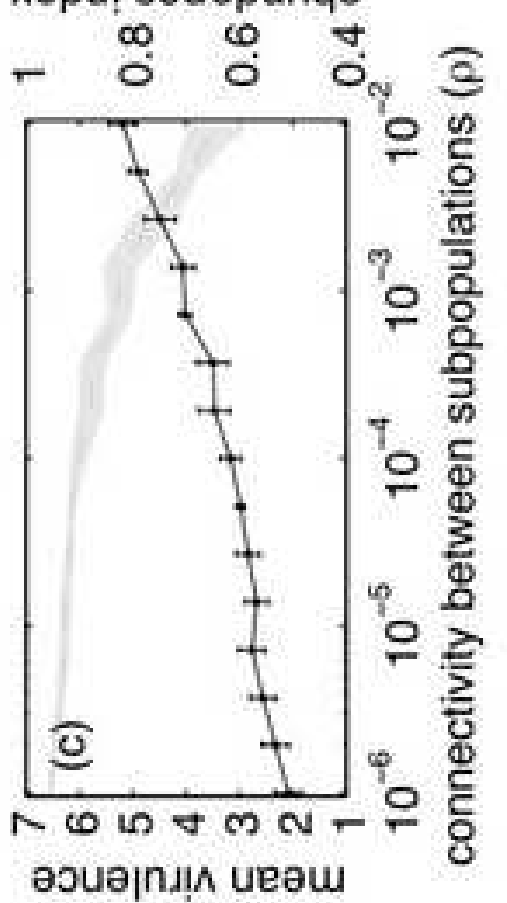

xәpu! әouepunqe

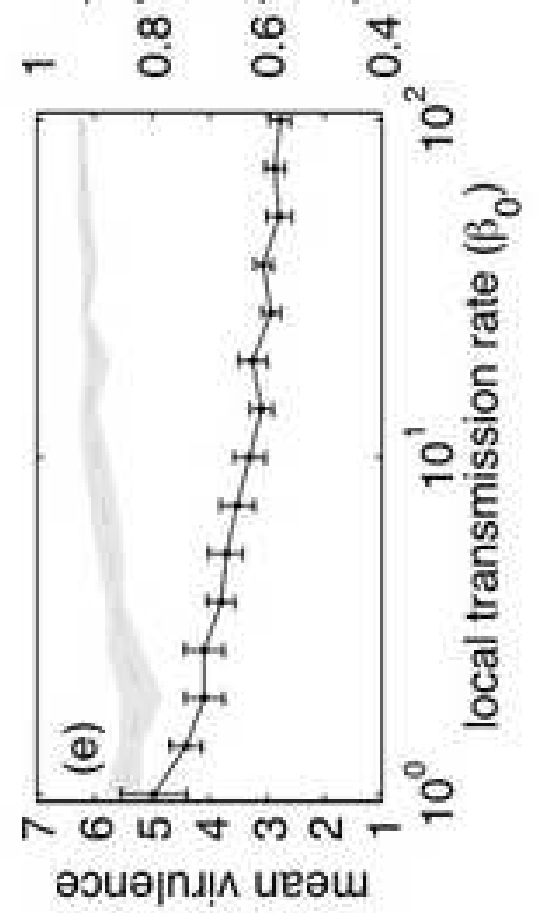




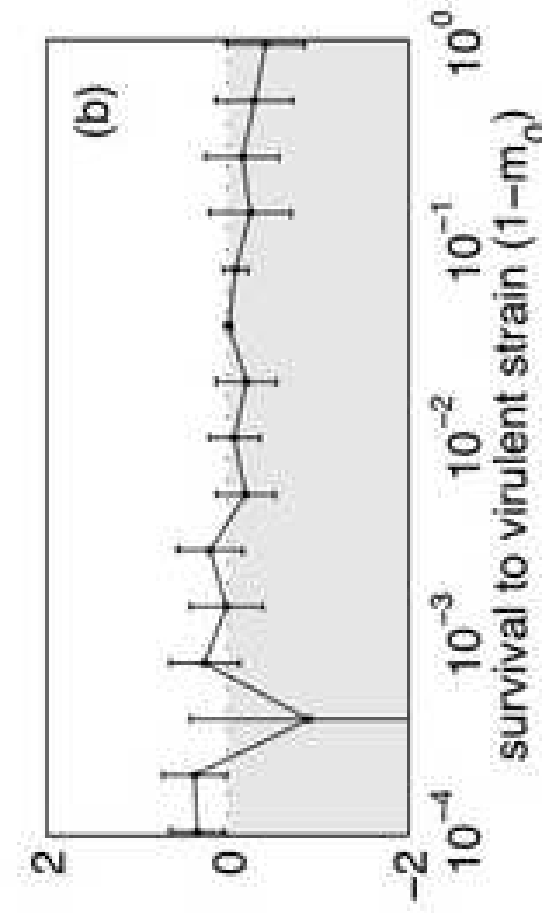

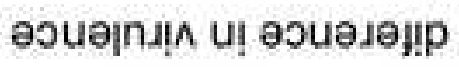

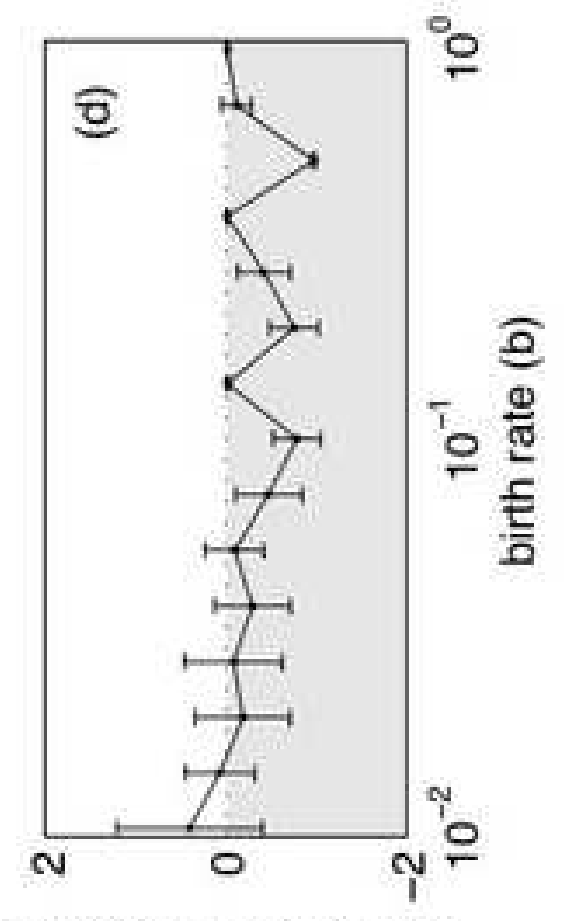

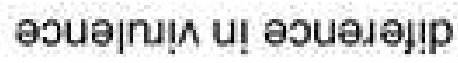

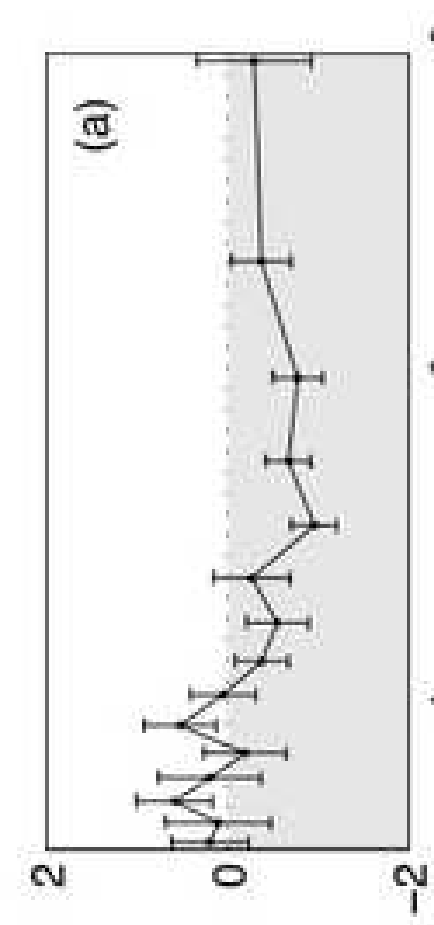

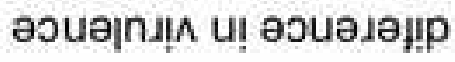

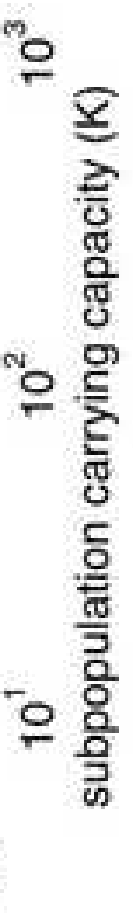
. 


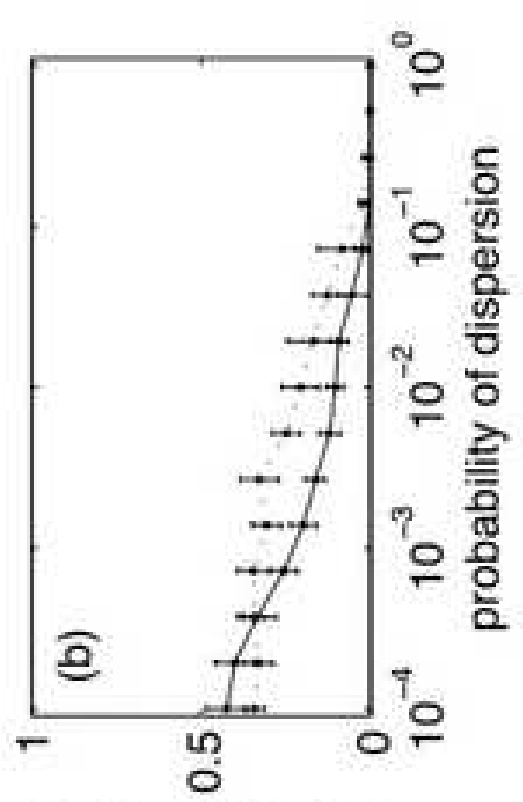

uํฺ̣ำx
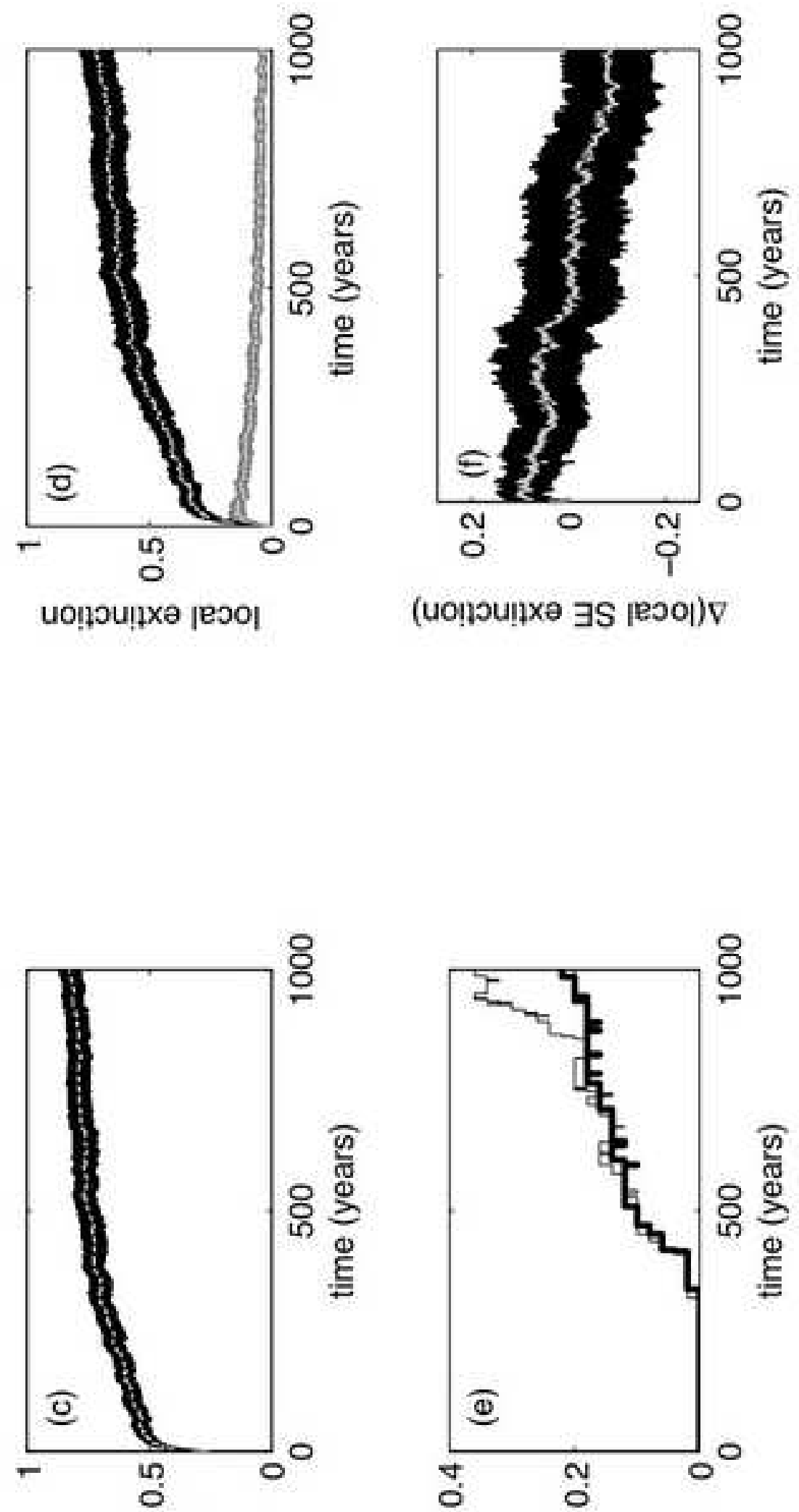

as to uoḷuododd

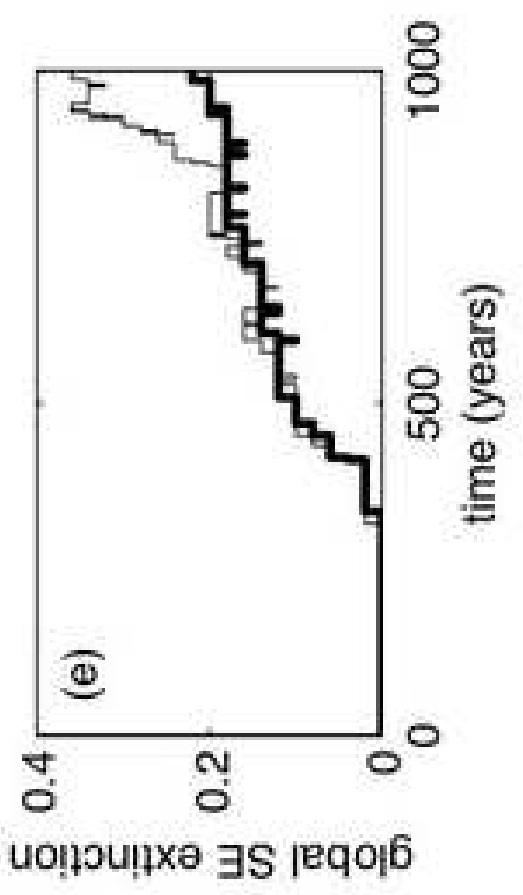




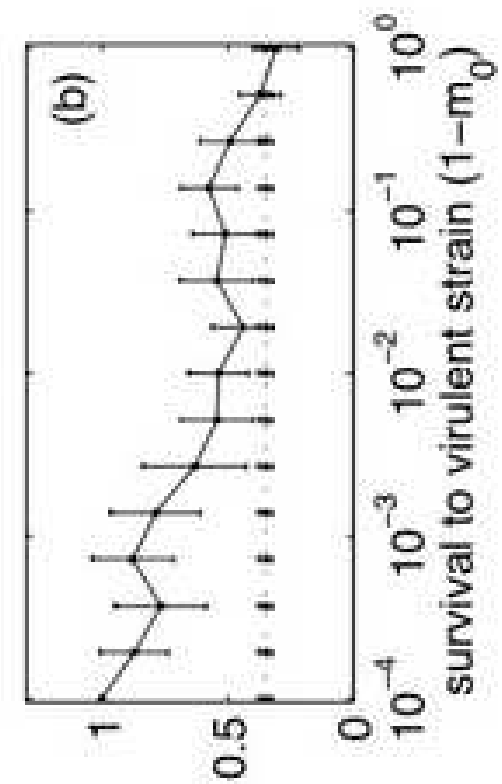

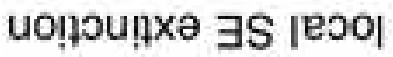

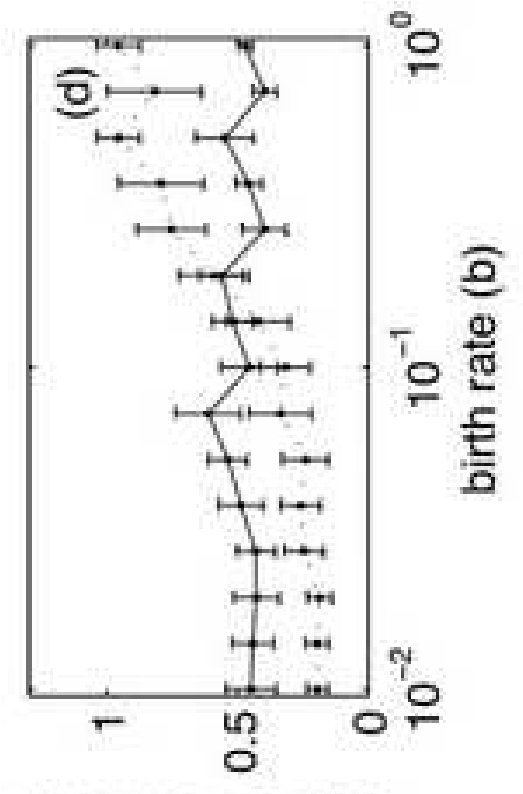

uo!̣ڤunxx $\exists \mathrm{S}$ ן

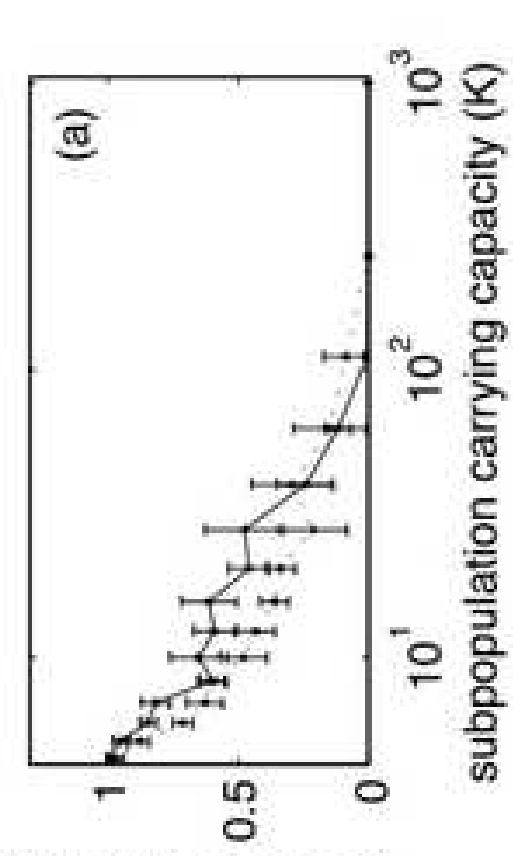

บо!ฺอน!ฺxə $\exists \mathrm{S}$ |еวо|

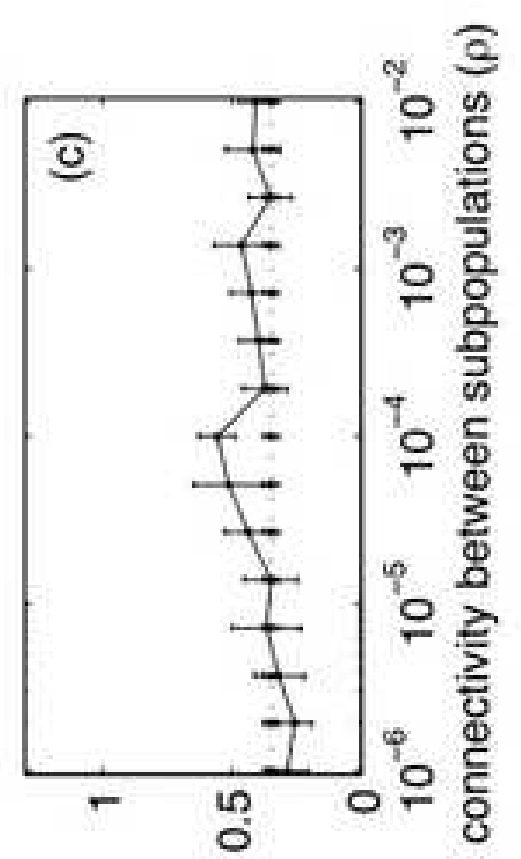

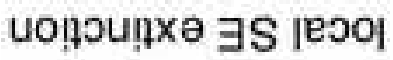

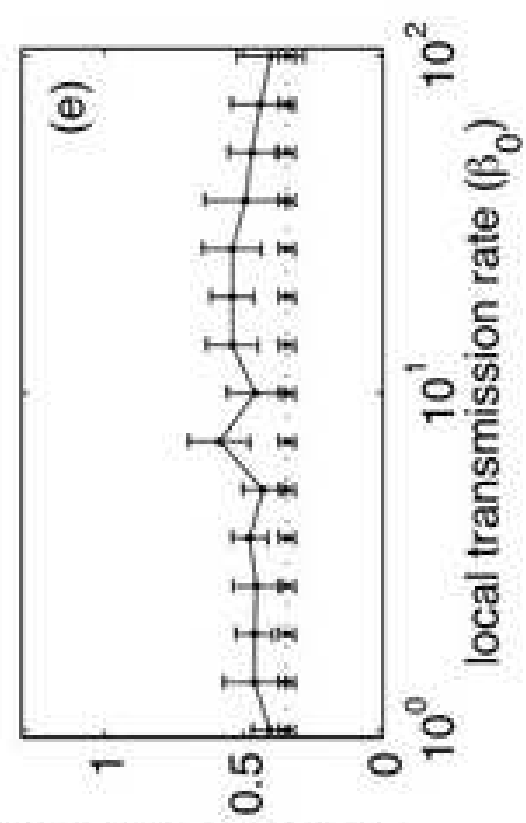

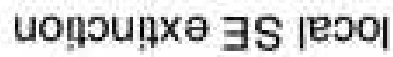

